\title{
Solution of inverse dynamics problems for contour error minimization in $\mathrm{CNC}$ machines
}

\author{
Charlie A. Ernesto • Rida T. Farouki
}

Received: 7 July 2009 / Accepted: 26 October 2009 / Published online: 13 November 2009

(C) The Author(s) 2009. This article is published with open access at Springerlink.com

\begin{abstract}
For CNC machines governed by typical feedback controllers, the problem of compensating for inertia and damping of the machine axes is solved by a priori modifications to the commanded path geometry. Standard second-order models of axis dynamics are expressed in terms of the path parameter $\xi$ rather than the time $t$ as independent variable, incurring ordinary differential equations with polynomial coefficients. For a commanded path specified as a Pythagorean-hodograph curve $\mathbf{R}(\xi)$ and a P controller, a modified path $\hat{\mathbf{R}}(\xi)$ can be determined as a rational Bézier curve, that precisely compensates for the axis inertia and damping, and thus (theoretically) achieves zero contour error. For PI, PID, or P-PI controllers, exact closed-form solutions for $\hat{\mathbf{R}}(\xi)$ are no longer possible, but polynomial approximations may be computed in the numerically stable Bernstein basis on $\xi \in[0,1]$. The inverse-dynamics path modification procedure is applicable to both constant feedrates and variable feedrates defined by polynomial functions $V(\xi)$ of the curve parameter. The method is described in the general context of PID controllers, and its implementation is then demonstrated for both P and PI controllers, governing motion along paths with extreme variations of curvature and/or parametric speed.
\end{abstract}

C. A. Ernesto · R. T. Farouki $(\varangle)$

Department of Mechanical and Aeronautical Engineering,

University of California, Davis, CA 95616, USA

e-mail: farouki@ucdavis.edu

C. A. Ernesto

e-mail: caernesto@ucdavis.edu
Keywords CNC machine - PID controller • Inverse dynamics $\cdot$ Feedrate $\cdot$ Contour error . Path modification - Pythagorean-hodograph curve

\section{Introduction}

CNC machines employ feedback control systems to independently drive each machine axis in order to achieve a given speed of the tool along a given path, relative to the workpiece. Due to the inherent machine/ controller dynamics, it is impossible to respond instantaneously to variations in commanded path geometry and speed. Consequently, the actual machine motion deviates from the desired motion in both path geometry (contour error) and speed along it (feedrate error). Contour error incurs appreciable inaccuracy of the machined part shape, but feedrate error has the lessserious consequence of altering the overall machining time. The focus of this study is on the "inverse dynamics" problem of identifying a priori modifications to the commanded path, for a given feedrate, that will cause the physical trajectory generated by the $\mathrm{CNC}$ machine to conform more closely to the original commanded path.

For brevity, we focus here on planar paths (the extension to spatial paths is elementary) and we consider only the intrinsic machine dynamics, without reference to cutting forces, external disturbances, etc. ${ }^{1}$

\footnotetext{
${ }^{1}$ The intrinsic machine dynamics may dominate in high-speed machining [4, 19, 21, 22] of complicated shapes, involving very large speeds and acceleration/deceleration rates.
} 
(one can, in principle, also compensate for such effects if quantitative models of them are available). A standard second-order model [26] for the axis dynamics is used, together with a P, PI, PID, or P-PI controller. For a commanded path specified as a parametric curve $\mathbf{R}(\xi)$, the independent variable in the differential equations governing the machine/controller dynamics is transformed from the time $t$ to the curve parameter $\xi$ in accordance with a given (possibly constant) feedrate function $V(\xi)$. By reverting these differential equations, a modified path $\hat{\mathbf{R}}(\xi)$ is sought, such that the actual executed path-under the influence of the machine/controller dynamics-agrees with the desired path $\mathbf{R}(\xi)$.

When expressed in terms of the curve parameter $\xi$, the machine/controller dynamical equations have non-constant coefficients. It is advantageous to use Pythagorean-hodograph ( $\mathrm{PH})$ curves [7] to specify the path $\mathbf{R}(\xi)$, because the coefficients of these differential equations are then polynomials in $\xi$. For a $\mathrm{P}$ controller, the modified path $\hat{\mathbf{R}}(\xi)$ can be exactly determined as a higher-order rational curve. For more sophisticated controllers, exact descriptions of $\hat{\mathbf{R}}(\xi)$ are no longer possible, but algorithms can be formulated to compute a convergent sequence of polynomial approximations to it.

The focus of this paper is the inverse dynamics pathmodification problem for smooth analytic curves. A number of authors have recently investigated pathmodification procedures for $\mathrm{CNC}$ machines, primarily to reduce contour error in the vicinity of sharp path corners through an "over-corner" approach $[5,17,18]$. These methods invoke an ad hoc replacement of sharp corners by smooth curve segments, empirically optimized to reduce contour error for a given corner angle, rather than solving an inverse dynamics problem.

The compensatory path modification scheme described herein is intended for off-line (rather than real-time) computation-the modified paths are precomputed, and then communicated as a modified part program to a $\mathrm{CNC}$ machine with a real-time interpolator capable of processing them. Also, the method is best suited to part programs that comprise relatively few analytic curve segments, rather than numerous short linear/circular $\mathrm{G}$ codes.

The plan for the remainder of this paper is as follows. Section 2 describes the basic machine/controller dynamic models employed herein, expressed in the time domain. These differential equations are transformed in Section 3 so that the time $t$ is replaced by the curve parameter $\xi$ as the independent variable, and the inverse dynamics path-modification problem is formulated through a reversion of the resulting equa- tions (which have non-constant coefficients). Section 4 presents an exact solution for the modified path in the case of a $\mathrm{P}$ controller, and an approximate solution (allowing refinement to any desired accuracy) in the case of a PI controller. Computed examples are presented to illustrate the ability of the a priori path modification procedure to minimize contour error along paths with strong curvature variations. Finally, Section 5 summarizes and assesses the results of the present study, and identifies other promising directions for further investigation.

\section{Machine/controller dynamics}

Let $(X(t), Y(t))$ denote the commanded path and $(x(t), y(t))$ the actual path executed by a CNC machine, both parameterized by time $t$. Standard models $[1,5]$ for the dynamics of CNC machines involve a determination of $(x(t), y(t))$ from $(X(t), Y(t))$ through differential equations of the form

$$
\begin{aligned}
& a_{x} \dddot{x}+b_{x} \ddot{x}+c_{x} \dot{x}+x=d_{x} \ddot{X}+e_{x} \dot{X}+X, \\
& a_{y} \dddot{y}+b_{y} \ddot{y}+c_{y} \dot{y}+y=d_{y} \ddot{Y}+e_{y} \dot{Y}+Y,
\end{aligned}
$$

where dots indicate time derivatives, and the constant coefficients $a_{x}, b_{x}, \ldots$ depend on the machine/ controller physical parameters. Figures 1 and 2 show block diagrams of typical physical systems giving rise to Eq. 1.

For brevity, we discuss only the $x$-axis dynamics: similar principles apply to the $y$-axis, with possibly different values for the physical parameters. The system variables (and their dimensions) are as follows- $u(\mathrm{~V})$ is the control variable; $k_{a}(\mathrm{~A} / \mathrm{V})$ and $k_{t}(\mathrm{Nm} / \mathrm{A})$ are the current amplifier and motor torque gains; $J\left(\mathrm{~kg} \mathrm{~m}^{2}\right)$ and $B\left(\mathrm{~kg} \mathrm{~m}^{2} / \mathrm{s}\right)$ are the $x$-axis inertia and viscous damping; $T(\mathrm{~N} \mathrm{~m}), \omega(\mathrm{rad} / \mathrm{s})$, and $\theta(\mathrm{rad})$ are the motor torque, angular speed, and position; and $r_{g}(\mathrm{~m} / \mathrm{rad})$ is the transmission ratio-i.e., the translation of the axis for unit rotation of the motor shaft.

We consider here several commonly used forms for the controller transfer function: PID control with proportional, integral, derivative gains $k_{p}, k_{i}$, and $k_{d}$ as shown in Fig. 1 (with $\mathrm{P}$ and PI control as the special cases $k_{i}=k_{d}=0$ and $\left.k_{d}=0\right)$ and P-PI control as employed in $[17,18]$. The latter, illustrated in Fig. 2, employs feedback of the motor angular speed $\omega$, as well as the axis position $x$. For brevity, we set $K=k_{a} k_{t} r_{g}$ henceforth, since the parameters $k_{a}, k_{t}$, and $r_{g}$ often occur in the form of this product. For each model, the system transfer function and the coefficients in the 


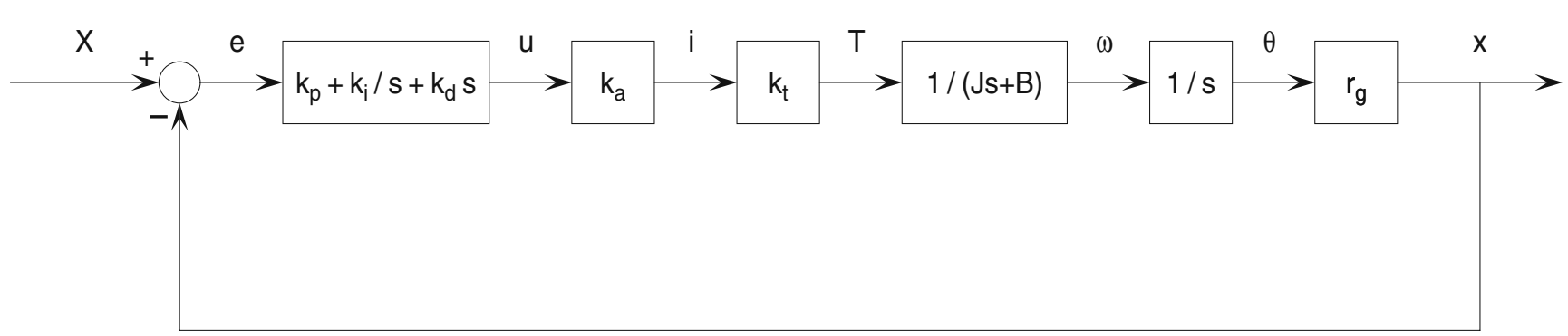

Fig. 1 Block diagram for the $x$-axis drive-the proportional, integral, and derivative gains of the PID controller are $k_{p}, k_{i}$, and $k_{d}$, while $e=X-x$ is the difference between the commanded and actual axis locations. The power amplifier converts the actuating signal $u$ into a current $i$ to the motor, which produces a

governing differential equations (Eq. 1) will be derived below.

\subsection{PID controller}

For the general PID controller in Fig. 1, the transfer function relating the Laplace transforms of the output $x$ and the input $X$ can be written as

$\frac{x}{X}=\frac{K\left(k_{d} s^{2}+k_{p} s+k_{i}\right)}{J s^{3}+\left(B+K k_{d}\right) s^{2}+K k_{p} s+K k_{i}}$.

This defines a third-order system, with three poles and two (real or complex conjugate) zeros. The coefficients of the differential equations (Eq. 1) are

$a_{x}=\frac{J}{K k_{i}}, \quad b_{x}=\frac{B+K k_{d}}{K k_{i}}, \quad c_{x}=\frac{k_{p}}{k_{i}}$,

$d_{x}=\frac{k_{d}}{k_{i}}, \quad e_{x}=\frac{k_{p}}{k_{i}}$.

Analogous results hold for the $y$-axis dynamics, using appropriate values for the physical parameters associated with that axis. torque $T$ that determines the angular speed $\omega$ through the system inertia $J$ and damping $B$. The motor shaft angle $\theta$, obtained by integration of $\omega$, determines the axis linear position $x$ through the transmission ratio $r_{g}$

\section{$2.2 \mathrm{P}$ controller}

As a special case of the PID controller shown in Fig. 1, consider the case of a simple $\mathrm{P}$ controller, specified by the choices $k_{i}=k_{d}=0$. The transfer function then reduces to

$\frac{x}{X}=\frac{K k_{p}}{J s^{2}+B s+K k_{p}}$.

This describes a second-order system, with two (real or complex conjugate) poles and no zeros. The differential equations (Eq. 1) then have the coefficients

$a_{x}=0, \quad b_{x}=\frac{J}{K k_{p}}, \quad c_{x}=\frac{B}{K k_{p}}, \quad d_{x}=0, \quad e_{x}=0$,

with analogous results for the $y$-axis dynamics.

\subsection{PI controller}

Since a P controller cannot ensure zero steady-state error, it is often desirable to upgrade to a PI controller (specified by $k_{d}=0$ in the PID controller). In this case, the transfer function becomes

$\frac{x}{X}=\frac{K\left(k_{p} s+k_{i}\right)}{J s^{3}+B s^{2}+K k_{p} s+K k_{i}}$.

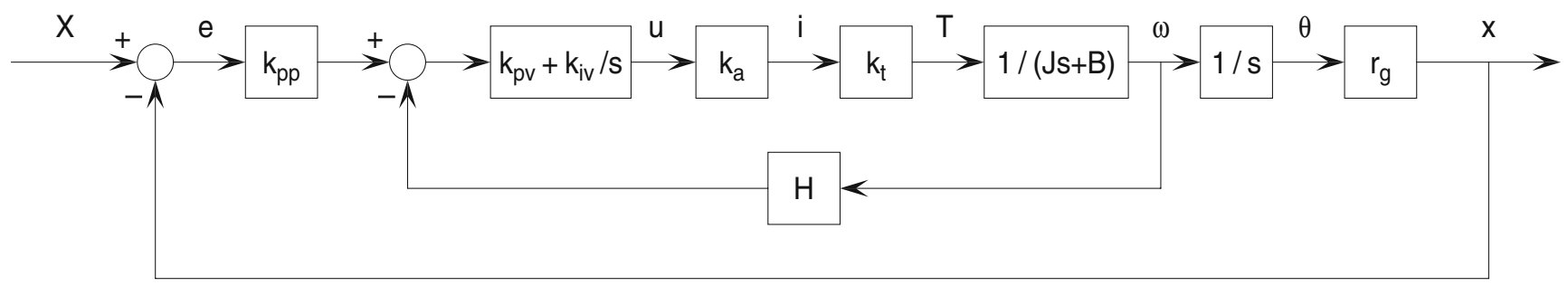

Fig. 2 Block diagram for $x$-axis drive with P-PI controller. The position loop is closed by the $\mathrm{P}$ controller, with proportional gain $k_{p p}$, and the velocity loop is closed by the PI controller, with proportional gain $k_{p v}$ and integral gain $k_{i v}$. The remainder of the block diagram is identical to that in Fig. 1 
This defines a third-order system, with three poles and one (real) zero. The differential equations (Eq. 1) then have the coefficients

$a_{x}=\frac{J}{K k_{i}}, \quad b_{x}=\frac{B}{K k_{i}}, \quad c_{x}=\frac{k_{p}}{k_{i}}, \quad d_{x}=0$,

$e_{x}=\frac{k_{p}}{k_{i}}$,

and analogous results hold for the $y$-axis dynamics.

\subsection{P-PI controller}

The P-PI controller, shown in Fig. 2, involves feedback of the position $x$ through a $\mathrm{P}$ controller, and the angular velocity $\omega$ through a PI controller, yielding the transfer function

$\frac{x}{X}=\frac{K k_{p p}\left(k_{p v} s+k_{i v}\right)}{J s^{3}+\left(B+H k_{a} k_{t} k_{p v}\right) s^{2}+k_{a} k_{t}\left(H k_{i v}+k_{p p} k_{p v} r_{g}\right) s+K k_{p p} k_{i v}}$.

This describes a third-order system with three poles and one real zero-the transfer function has the same form as the PI controller, but the coefficients (and the physical parameters that determine them) differ. In the differential equations (Eq. 1), the coefficients are

$a_{x}=\frac{J}{K k_{p p} k_{i v}}, \quad b_{x}=\frac{B+H k_{a} k_{t} k_{p v}}{K k_{p p} k_{i v}}$,

$c_{x}=\frac{k_{p v}}{k_{i v}}+\frac{H}{k_{p p} r_{g}}, \quad d_{x}=0, \quad e_{x}=\frac{k_{p v}}{k_{i v}}$,

with analogous results for the $y$-axis dynamics.

\section{Modelling in curve parameter domain}

The time domain is conventionally used to study machine dynamics, but in practice the desired path $\mathbf{R}(\xi)=$ $(X(\xi), Y(\xi))$ - generated by a CAD/CAM system-is not parameterized by time, but rather by polynomial or rational functions of a general parameter $\xi$. The parametric speed

$\sigma(\xi)=\left|\mathbf{R}^{\prime}(\xi)\right|=\sqrt{X^{\prime 2}(\xi)+Y^{\prime 2}(\xi)}=\frac{\mathrm{d} s}{\mathrm{~d} \xi}$

of $\mathbf{R}(\xi)$ is the function specifying the rate of change of its arc length $s$ with respect to the parameter $\xi$. Ideally, one would like to have $\sigma \equiv 1$ (i.e., $s \equiv \xi$ ), but the only curves that admit parameterization by rational functions of their arc length are straight lines $[12,13]$. For the PH curves, $\sigma(\xi)$ is a polynomial function of the curve parameter [7].

For general parametric curves, the (constant or variable) speed or feedrate $V=\mathrm{d} s / \mathrm{d} t$ must be specified along the path to fix the relation between the curve parameter $\xi$ and elapsed time $t$. If the curve is regulari.e., it satisfies $\sigma(\xi) \neq 0$ for all $\xi$-and the feedrate $V$ is everywhere positive, there will be a one-to-one relation between the variables $\xi, s$, and $t$ describing progress along the path, but it is the curve parameter $\xi$ that the real-time interpolator algorithm employs to compute path reference points $\left(X_{k}, Y_{k}\right)=\left(X\left(\xi_{k}\right), Y\left(\xi_{k}\right)\right)$ at each servo sampling instant $k \Delta t, k=0,1,2, \ldots[14,15,20$, $24,25]$.

\subsection{Transformation of equations}

To formulate the inverse dynamics problem, Eq. 1 is re-cast in terms of the parameter $\xi$, rather than time $t$, as the independent variable. This is accomplished by invoking the chain rule to observe that derivatives with respect to $t$ and $\xi$ are related by

$\frac{\mathrm{d}}{\mathrm{d} t}=\frac{\mathrm{d} s}{\mathrm{~d} t} \frac{\mathrm{d} \xi}{\mathrm{d} s} \frac{\mathrm{d}}{\mathrm{d} \xi}=\frac{V}{\sigma} \frac{\mathrm{d}}{\mathrm{d} \xi}$.

Suppose that, for any given path and feedrate, the function $\xi(t)$ specifies the variation of the curve parameter with time. Applying Eq. 2 repeatedly, we can express the derivatives of $\xi(t)$ recursively ${ }^{2}$ as

$$
\begin{aligned}
& \dot{\xi}=\frac{V}{\sigma}, \quad \ddot{\xi}=\frac{\sigma V^{\prime}-\sigma^{\prime} V}{\sigma^{2}} \dot{\xi}, \\
& \dddot{\xi}=\frac{\sigma V^{\prime}-3 \sigma^{\prime} V}{\sigma^{2}} \ddot{\xi}+\frac{\sigma V^{\prime \prime}-\sigma^{\prime \prime} V}{\sigma^{2}} \dot{\xi}^{2}, \quad \text { etc. },
\end{aligned}
$$

where primes indicate derivatives with respect to $\xi$. The parametric speed $\sigma$ and its derivatives, required in Eq. 3, can also be expressed recursively as

$$
\begin{aligned}
\sigma & =\left|\mathbf{R}^{\prime}\right|, \quad \sigma^{\prime}=\frac{\mathbf{R}^{\prime} \cdot \mathbf{R}^{\prime \prime}}{\sigma}, \\
\sigma^{\prime \prime} & =\frac{\mathbf{R}^{\prime} \cdot \mathbf{R}^{\prime \prime \prime}+\left|\mathbf{R}^{\prime \prime}\right|^{2}-\sigma^{\prime 2}}{\sigma}, \quad \text { etc. }
\end{aligned}
$$

\footnotetext{
${ }^{2}$ These relations were first developed in [15], in the context of formulating real-time $\mathrm{CNC}$ interpolators for variable feedrate functions.
} 
In Eq. 3, the feedrate is assumed to be specified as a function $V(\xi)$ of the curve parameter (if $V=$ constant, we have $V^{\prime}=V^{\prime \prime}=\cdots=0$ ). Since $\sigma, \sigma^{\prime}, \sigma^{\prime \prime}, \ldots$ are known functions of $\xi$, the expressions in Eq. 3 are all known functions of $\xi$.

Writing $V / \sigma$ in Eq. 2 as $\dot{\xi}$ and applying it repeatedly, time derivatives can be converted to $\xi$ derivatives through

$$
\begin{aligned}
\frac{\mathrm{d}}{\mathrm{d} t} & =\dot{\xi} \frac{\mathrm{d}}{\mathrm{d} \xi}, \quad \frac{\mathrm{d}^{2}}{\mathrm{~d} t^{2}}=\dot{\xi}^{2} \frac{\mathrm{d}^{2}}{\mathrm{~d} \xi^{2}}+\ddot{\xi} \frac{\mathrm{d}}{\mathrm{d} \xi}, \\
\frac{\mathrm{d}^{3}}{\mathrm{~d} t^{3}} & =\dot{\xi}^{3} \frac{\mathrm{d}^{3}}{\mathrm{~d} \xi^{3}}+3 \dot{\xi} \ddot{\xi} \frac{\mathrm{d}^{2}}{\mathrm{~d} \xi^{2}}+\dddot{\xi} \frac{\mathrm{d}}{\mathrm{d} \xi}, \quad \text { etc. }
\end{aligned}
$$

Transforming the differential equations (Eq. 1) in this manner, we obtain

$$
\begin{aligned}
& a_{x} \dot{\xi}^{3} x^{\prime \prime \prime}+\left(3 a_{x} \ddot{\xi}+b_{x} \dot{\xi}\right) \dot{\xi} x^{\prime \prime}+\left(a_{x} \dddot{\xi}+b_{x} \ddot{\xi}+c_{x} \dot{\xi}\right) x^{\prime}+x \\
& \quad=d_{x} \dot{\xi}^{2} X^{\prime \prime}+\left(d_{x} \ddot{\xi}+e_{x} \dot{\xi}\right) X^{\prime}+X, \\
& a_{y} \dot{\xi}^{3} y^{\prime \prime \prime}+\left(3 a_{y} \ddot{\xi}+b_{y} \dot{\xi}\right) \dot{\xi} y^{\prime \prime}+\left(a_{y} \dddot{\xi}+b_{y} \ddot{\xi}+c_{y} \dot{\xi}\right) y^{\prime}+y \\
& \quad=d_{y} \dot{\xi}^{2} Y^{\prime \prime}+\left(d_{y} \ddot{\xi}+e_{y} \dot{\xi}\right) Y^{\prime}+Y,
\end{aligned}
$$

where again primes indicate derivatives with respect to $\xi$. Substituting from Eq. 3 and multiplying through by $\sigma^{5}$, these equations can be written as

$$
\begin{aligned}
\alpha_{x} x^{\prime \prime \prime}+\beta_{x} x^{\prime \prime}+\gamma_{x} x^{\prime}+\delta_{x} x & =\lambda_{x} X^{\prime \prime}+\mu_{x} X^{\prime}+v_{x} X, \\
\alpha_{y} y^{\prime \prime \prime}+\beta_{y} y^{\prime \prime}+\gamma_{y} y^{\prime}+\delta_{y} y & =\lambda_{y} Y^{\prime \prime}+\mu_{y} Y^{\prime}+v_{y} Y,
\end{aligned}
$$

where $\alpha_{x}, \beta_{x}, \ldots$ are functions of $\xi$, defined by

$$
\begin{aligned}
\alpha_{x}= & a_{x} \sigma^{2} V^{3}, \quad \alpha_{y}=a_{y} \sigma^{2} V^{3}, \\
\beta_{x}= & \sigma V^{2}\left[3 a_{x}\left(\sigma V^{\prime}-\sigma^{\prime} V\right)+b_{x} \sigma^{2}\right], \\
\beta_{y}= & \sigma V^{2}\left[3 a_{y}\left(\sigma V^{\prime}-\sigma^{\prime} V\right)+b_{y} \sigma^{2}\right], \\
\gamma_{x}=V\left[\left(a_{x}\left(\sigma V^{\prime}-3 \sigma^{\prime} V\right)+b_{x} \sigma^{2}\right)\left(\sigma V^{\prime}-\sigma^{\prime} V\right)\right. & \\
& \left.\quad+a_{x} \sigma V\left(\sigma V^{\prime \prime}-\sigma^{\prime \prime} V\right)+c_{x} \sigma^{4}\right], \\
\gamma_{y}=V & {\left[\left(a_{y}\left(\sigma V^{\prime}-3 \sigma^{\prime} V\right)+b_{y} \sigma^{2}\right)\left(\sigma V^{\prime}-\sigma^{\prime} V\right)\right.} \\
& \left.\quad+a_{y} \sigma V\left(\sigma V^{\prime \prime}-\sigma^{\prime \prime} V\right)+c_{y} \sigma^{4}\right], \\
\delta_{x}= & \delta_{y}=v_{x}=v_{y}=\sigma^{5}, \\
\lambda_{x}= & d_{x} \sigma^{3} V^{2}, \quad \lambda_{y}=d_{y} \sigma^{3} V^{2}, \\
\mu_{x}= & \sigma^{2} V\left[d_{x}\left(\sigma V^{\prime}-\sigma^{\prime} V\right)+e_{x} \sigma^{2}\right], \\
\mu_{y}= & \sigma^{2} V\left[d_{y}\left(\sigma V^{\prime}-\sigma^{\prime} V\right)+e_{y} \sigma^{2}\right] .
\end{aligned}
$$

If the commanded path $\mathbf{R}(\xi)=(X(\xi), Y(\xi))$ is a $\mathrm{PH}$ curve, so that $\sigma(\xi)$ is a polynomial, and the feedrate is a specified polynomial function $V(\xi)$ of the curve parameter, the executed path $\mathbf{r}(\xi)=(x(\xi), y(\xi))$ is the solution of the differential equations (Eq. 5), with the polynomials in $\xi$ (Eq. 6) as coefficients.

\subsection{Inverse dynamics problem}

Instead of computing the actual path from the commanded path, however, we wish to use Eq. 5 to solve an "inverse" problem-we seek a modified commanded path $(\hat{X}(\xi), \hat{Y}(\xi))$ that, subject to the machine dynamics, yields an executed path exactly coincident with the original commanded path, i.e., $(x(\xi), y(\xi)) \equiv(X(\xi), Y(\xi))$. Substituting $(\hat{X}(\xi), \hat{Y}(\xi))$ for $(X(\xi), Y(\xi))$ and $(X(\xi), Y(\xi))$ for $(x(\xi), y(\xi))$ in Eq. 5, we obtain

$$
\begin{aligned}
\lambda_{x} \hat{X}^{\prime \prime}+\mu_{x} \hat{X}^{\prime}+v_{x} \hat{X} & =\alpha_{x} X^{\prime \prime \prime}+\beta_{x} X^{\prime \prime}+\gamma_{x} X^{\prime}+\delta_{x} X \\
\lambda_{y} \hat{Y}^{\prime \prime}+\mu_{y} \hat{Y}^{\prime}+v_{y} \hat{Y} & =\alpha_{y} Y^{\prime \prime \prime}+\beta_{y} Y^{\prime \prime}+\gamma_{y} Y^{\prime}+\delta_{y} Y .
\end{aligned}
$$

These are linear differential equations for $\hat{X}(\xi), \hat{Y}(\xi)$ in which the coefficients and right-hand sides are known polynomial functions of $\xi$. We are interested in finding solutions to these equations over the parameter domain $\xi \in[0,1]$.

In the case of a $\mathrm{P}$ controller, we have $d_{x}=e_{x}=d_{y}=$ $e_{y}=0$ and, hence, $\lambda_{x}(\xi)=\mu_{x}(\xi)=\lambda_{y}(\xi)=\mu_{y}(\xi) \equiv 0$, so $\hat{X}(\xi), \hat{Y}(\xi)$ can be determined exactly from Eq. 7 as rational functions (see Section 4.1). For PI control, $d_{x}=d_{y}=0$ so $\lambda_{x}(\xi)=\lambda_{y}(\xi) \equiv 0$ and Eq. 7 are firstorder differential equations for $\hat{X}(\xi), \hat{Y}(\xi)$ requiring an initial condition for a unique solution. Finally, for PID control, all the left-hand-side coefficients in Eq. 7 are non-vanishing, and hence they are second-order equations, requiring two initial conditions for a unique solution. Note that, since $\sigma(\xi)$ and $V(\xi)$ are positive for all $\xi$, the differential equations (Eq. 1) have no singular points-i.e., there are no real values of $\xi$ at which the coefficient of the highest-order term vanishes [2].

In general, linear differential equations with polynomial coefficients do not admit "simple" (polynomial or rational) solutions: see the Appendix. As an alternative, one may seek infinite power series solutionsbut the truncation error and radius of convergence is often difficult to assess. In the case of PI or PID control, we therefore seek approximate polynomial solutions to the equations, represented in the numerically stable Bernstein basis $[8,10,11]$.

If the path $\mathbf{R}(\xi)=(X(\xi), Y(\xi))$ is a $\mathrm{PH}$ curve of (odd) degree $n$, and the feedrate function $V(\xi)$ is a 
polynomial of degree $m$, we have $\operatorname{deg}(\sigma)=n-1$ and (assuming for simplicity that $m<n$ ):

$$
\begin{aligned}
& \operatorname{deg}\left(\alpha_{x}, \alpha_{y}\right)=3 m+2 n-2, \\
& \operatorname{deg}\left(\beta_{x}, \beta_{y}\right)=\operatorname{deg}\left(\lambda_{x}, \lambda_{y}\right)=2 m+3 n-3, \\
& \operatorname{deg}\left(\gamma_{x}, \gamma_{y}\right)=\operatorname{deg}\left(\mu_{x}, \mu_{y}\right)=m+4 n-4, \\
& \operatorname{deg}\left(\delta_{x}, \delta_{y}\right)=\operatorname{deg}\left(v_{x}, v_{y}\right)=5 n-5 .
\end{aligned}
$$

All the coefficients (Eq. 6) are then of degree $\leq 5 n-5$, and the right-hand sides in Eq. 7 are known polynomials of degree $6 n-5$ in $\xi$, that can be expressed [23] in the numerically stable Bernstein basis on $\xi \in[0,1]$.

\subsection{Interpretation of modified path}

Before proceeding to a detailed description of the path modification and the "induced feedrate" associated with it, one should have a clear picture of their significance and their role in compensating for the machine dynamics.

For any given commanded path $\mathbf{R}(\xi)=(X(\xi), Y(\xi))$ and feedrate $V(\xi)$, let $\hat{\mathbf{R}}(\xi)=(\hat{X}(\xi), \hat{Y}(\xi))$ be the modified path, obtained by solving Eq. 7. For each $\xi \in$ $[0,1]$, the points $\mathbf{R}(\xi)$ and $\hat{\mathbf{R}}(\xi)$ of the original and modified paths correspond to the same instant in time, namely,

$t(\xi)=\int_{0}^{\xi} \frac{\sigma}{V} \mathrm{~d} \xi$.

Note, in particular, that the total traversal time $T=t(1)$ is the same for the modified path $\hat{\mathbf{R}}(\xi)$ as for the original path $\mathbf{R}(\xi)$.

When using the modified path, the real-time interpolator should compute reference-point parameter values $\xi_{1}, \xi_{2}, \xi_{3}, \ldots$ corresponding to the sampling times $\Delta t, 2 \Delta t, 3 \Delta t, \ldots$ based upon the original commanded path $\mathbf{R}(\xi)$, its parametric speed $\sigma(\xi)$, and the original feedrate function $V(\xi)$. The output of the real-time interpolator, however, will be the sequence of reference points $\hat{\mathbf{R}}\left(\xi_{1}\right), \hat{\mathbf{R}}\left(\xi_{2}\right), \hat{\mathbf{R}}\left(\xi_{3}\right), \ldots$ on the modified pathrather than the points $\mathbf{R}\left(\xi_{1}\right), \mathbf{R}\left(\xi_{2}\right), \mathbf{R}\left(\xi_{3}\right), \ldots$ on the original commanded path. In other words, the real-time interpolator algorithm is modified only in the final step, to compute the reference points from $\xi_{1}, \xi_{2}, \xi_{3}, \ldots$ using $\hat{\mathbf{R}}(\xi)$ instead of $\mathbf{R}(\xi)$.

The parametric speed $\hat{\sigma}(\xi)$ and cumulative arc length $\hat{s}(\xi)$ of the modified path $\hat{\mathbf{R}}(\xi)$ are defined by

$\hat{\sigma}(\xi)=|\hat{\mathbf{R}}(\xi)|=\sqrt{\hat{X}^{\prime 2}(\xi)+\hat{Y}^{\prime 2}(\xi)}$

and

$\hat{s}(\xi)=\int_{0}^{\xi} \hat{\sigma} \mathrm{d} \xi$.
Now the modified path $\hat{\mathbf{R}}(\xi)$ and time function (Eq. 8) define an induced feedrate $\hat{V}(\xi)$ that differs from the feedrate $V(\xi)$ specified for the original path $\mathbf{R}(\xi)$. This can be seen by noting that

$V=\sigma \frac{\mathrm{d} \xi}{\mathrm{d} t} \quad$ and $\quad \hat{V}=\hat{\sigma} \frac{\mathrm{d} \xi}{\mathrm{d} t}$,

where the function $\xi(t)$ in Eq. 9 is unique-it identifies corresponding points on $\mathbf{R}(\xi)$ and $\hat{\mathbf{R}}(\xi)$ at each time $t$. Since $\sigma(\xi)$ and $\hat{\sigma}(\xi)$ are in general different, $\hat{V}(\xi)$ and $V(\xi)$ also differ: from Eq. 9, we see that $\hat{V} / V=\hat{\sigma} / \sigma$. Note, in particular, that a constant feedrate $V$ specified for $\mathbf{R}(\xi)$ will generally incur a variable induced feedrate $\hat{V}$ along the modified path $\hat{\mathbf{R}}(\xi)$.

There is no need to explicitly compute the induced feedrate: the real-time interpolator algorithm generates it "implicitly" by a non-uniform spacing of the reference points $\hat{\mathbf{R}}\left(\xi_{1}\right), \hat{\mathbf{R}}\left(\xi_{2}\right), \hat{\mathbf{R}}\left(\xi_{3}\right), \ldots$ at sampling times $\Delta t, 2 \Delta t, 3 \Delta t, \ldots$ on the modified path. In the examples presented below, we shall see that the modified path often exhibits tight "loops" with corresponding "spikes" in the induced feedrate. This extreme behavior in the commanded motion is necessary to overcome the inherent smoothing effects of the machine inertia and damping. In combination, the modified path $\hat{\mathbf{R}}(\xi)$ and induced feedrate $\hat{V}(\xi)$ compensate for the machine/controller dynamics, to ensure a physical output motion corresponding to accurate traversal of the original commanded path $\mathbf{R}(\xi)$ at the original commanded feedrate $V(\xi)$.

\section{Path modification procedure}

We now discuss implementation of the path modification procedure, for the case of $\mathrm{P}$ and PI controllers. The $\mathrm{P}$ controller permits exact definition of the modified path as a rational Bézier curve. For the PI controller, polynomial approximations to the modified path are computed. Since the P-PI controller is functionally equivalent to the PI controller, we shall not consider it further here. Although the path modification procedure for a PID controller is not more complicated than for a PI controller in basic methodology (only in the implementation details), we omit full treatment of it at present.

To compare machine performance in response to the original commanded path $\mathbf{R}(\xi)$ and the modified path $\hat{\mathbf{R}}(\xi)$ as input, the machine dynamics were simulated in MATLAB using the Isim function. As input to lsim, a sequence of reference points (corresponding to time increments equal to the sampling interval $\Delta t$ ) was computed by a real-time interpolator algorithm, 
according to the prescribed path and feedrate. In the simulations, a nominal sampling frequency of $f=$ $1 \mathrm{kHz}$ was employed, corresponding to a sampling interval of $\Delta t=0.001 \mathrm{~s}$. For cases where a constant feedrate $V$ was employed with a P controller, the simulation was started at time $t=-T$, so the output during the interval $t \in[0, T]$ is representative of the steadystate behavior.

\section{1 $\mathrm{P}$ controller}

In the case of a P controller, we have $a_{x}=d_{x}=e_{x}=0$ and $a_{y}=d_{y}=e_{y}=0$, and hence the coefficients (Eq. 6) reduce to

$\alpha_{x}=\alpha_{y}=\lambda_{x}=\lambda_{y}=\mu_{x}=\mu_{y}=0$,

$\beta_{x}=b_{x} \sigma^{3} V^{2}, \quad \beta_{y}=b_{y} \sigma^{3} V^{2}$,

$\gamma_{x}=\sigma^{2} V\left[b_{x}\left(\sigma V^{\prime}-\sigma^{\prime} V\right)+c_{x} \sigma^{2}\right]$,

$\gamma_{y}=\sigma^{2} V\left[b_{y}\left(\sigma V^{\prime}-\sigma^{\prime} V\right)+c_{y} \sigma^{2}\right]$,

$\delta_{x}=\delta_{y}=v_{x}=v_{y}=\sigma^{5}$.

Canceling out the common factor $\sigma^{2}$, Eq. 7 then becomes

$\sigma^{3} \hat{X}=b_{x} \sigma V^{2} X^{\prime \prime}+V\left[b_{x}\left(\sigma V^{\prime}-\sigma^{\prime} V\right)+c_{x} \sigma^{2}\right] X^{\prime}+\sigma^{3} X$, $\sigma^{3} \hat{Y}=b_{y} \sigma V^{2} Y^{\prime \prime}+V\left[b_{y}\left(\sigma V^{\prime}-\sigma^{\prime} V\right)+c_{y} \sigma^{2}\right] Y^{\prime}+\sigma^{3} Y$.

This allows us to determine the modified path explicitly as

$$
\begin{aligned}
& \hat{X}=\frac{b_{x} \sigma V^{2} X^{\prime \prime}+V\left[b_{x}\left(\sigma V^{\prime}-\sigma^{\prime} V\right)+c_{x} \sigma^{2}\right] X^{\prime}+\sigma^{3} X}{\sigma^{3}}, \\
& \hat{Y}=\frac{b_{y} \sigma V^{2} Y^{\prime \prime}+V\left[b_{y}\left(\sigma V^{\prime}-\sigma^{\prime} V\right)+c_{y} \sigma^{2}\right] Y^{\prime}+\sigma^{3} Y}{\sigma^{3}} .
\end{aligned}
$$

If $\mathbf{R}(\xi)=\left(X(\xi), Y(\xi)\right.$ is a $\mathrm{PH}$ curve $^{3}$ of degree $n$, and the feedrate $V(\xi)$ is of degree $<n$, expression 11 specifies the modified path $\hat{\mathbf{R}}(\xi)=(\hat{X}(\xi), \hat{Y}(\xi))$ as a rational curve of degree $4 n-3$. When $\mathbf{R}(\xi)$ is a $\mathrm{PH}$ quintic, for example, $\hat{\mathbf{R}}(\xi)$ can be exactly represented as a degree 17 rational Bézier curve-whose control points and weights can be readily derived from Eq. 11.

\footnotetext{
${ }^{3}$ Although we emphasize the PH curves here on account of their flexible and essentially exact real-time interpolator algorithms, and the desire to obtain a rational modified curve $\hat{\mathbf{R}}(\xi)$, it should be noted that expression 11 applies to any analytic path $\mathbf{R}(\xi)$ for which we can compute the parametric speed $\sigma=\left|\mathbf{R}^{\prime}\right|$ and its derivative $\sigma^{\prime}$.
}

It is more convenient to regard the modified path $\hat{\mathbf{R}}(\xi)$ as a superposition

$\hat{\mathbf{R}}(\xi)=\mathbf{R}(\xi)+\Delta \mathbf{R}(\xi)$

of the original path $\mathbf{R}(\xi)$ and a vector displacement $\Delta \mathbf{R}(\xi)=(\Delta X(\xi), \Delta Y(\xi))$, whose components are defined by

$$
\begin{aligned}
& \Delta X=b_{x}(V / \sigma)^{2} X^{\prime \prime}+(V / \sigma)\left[b_{x}(V / \sigma)^{\prime}+c_{x}\right] X^{\prime}, \\
& \Delta Y=b_{y}(V / \sigma)^{2} Y^{\prime \prime}+(V / \sigma)\left[b_{y}(V / \sigma)^{\prime}+c_{y}\right] Y^{\prime},
\end{aligned}
$$

with $(V / \sigma)^{\prime}=\left(\sigma V^{\prime}-\sigma^{\prime} V\right) / \sigma^{2}$. Expression 12 offers a more efficient means of evaluating the modified path than explicit representation as a high-degree rational Bézier curve. In general, the displacement $\Delta \mathbf{R}(\xi)$ will vary in both magnitude and direction along the original path $\mathbf{R}(\xi)$. Note that for identical axes- $b_{x}=b_{y}(=$ $b$, say) and $c_{x}=c_{y}(=c$, say) - it can be expressed as

$$
\Delta \mathbf{R}(\xi)=b \dot{\xi}^{2} \mathbf{R}^{\prime \prime}(\xi)+(b \ddot{\xi}+c \dot{\xi}) \mathbf{R}^{\prime}(\xi),
$$

where $\dot{\xi}$ and $\ddot{\xi}$ are given as functions of $\xi$ by Eq. 3 .

The simplest case is that of a constant feedrate $V$, i.e., $V^{\prime} \equiv 0$. It should be noted that, in this case, the expression for $\Delta \mathbf{R}(\xi)=(\Delta X(\xi), \Delta Y(\xi))$ is non-vanishing at $\xi=0$ and 1, i.e., there is a discrepancy between the start and end points of the original path $\mathbf{R}(\xi)$ and the modified path $\hat{\mathbf{R}}(\xi)$. This is a consequence of the fact that the constant feedrate $V$ applies to the entire curve, $-\infty<\xi<+\infty$, not just $\xi \in[0,1]$. Finite displacements at $\xi=0$ and 1 are needed to compensate for the machine/controller dynamics on passing through those points with the constant speed $V$. To obtain a modified path satisfying $\hat{\mathbf{R}}(0)=\mathbf{R}(0)$ and $\hat{\mathbf{R}}(1)=\mathbf{R}(1)$, one must use a feedrate function $V(\xi)$ with $V(0)=$ $V(1)=0$, i.e., the motion must start and end at rest.

Example 1 As test curve for the case of a $\mathrm{P}$ controller, we use the PH quintic interpolant [9] to the Hermite data (with dimensions in meters):

$\begin{array}{ll}\mathbf{r}(0)=(0.0,0.0), & \mathbf{r}^{\prime}(0)=(3.0,2.5), \\ \mathbf{r}(1)=(0.7,0.1), & \mathbf{r}^{\prime}(1)=(2.5,-3.0),\end{array}$

shown in Fig. 3. In terms of the complex model [6] for planar PH curves, the hodograph $\mathbf{r}^{\prime}(\xi)=\left[\mathbf{w}_{0}(1-\xi)^{2}+\right.$ $\left.\mathbf{w}_{1} 2(1-\xi) \xi+\mathbf{w}_{2} \xi^{2}\right]^{2}$ of this curve is the square of the complex quadratic polynomial with Bernstein coefficients

$$
\begin{aligned}
& \mathbf{w}_{0}=1.85810721+0.67272760 \mathrm{i}, \\
& \mathbf{w}_{1}=-1.11728797+0.43234437 \mathrm{i}, \\
& \mathbf{w}_{2}=1.78957046-0.83818997 \mathrm{i} .
\end{aligned}
$$



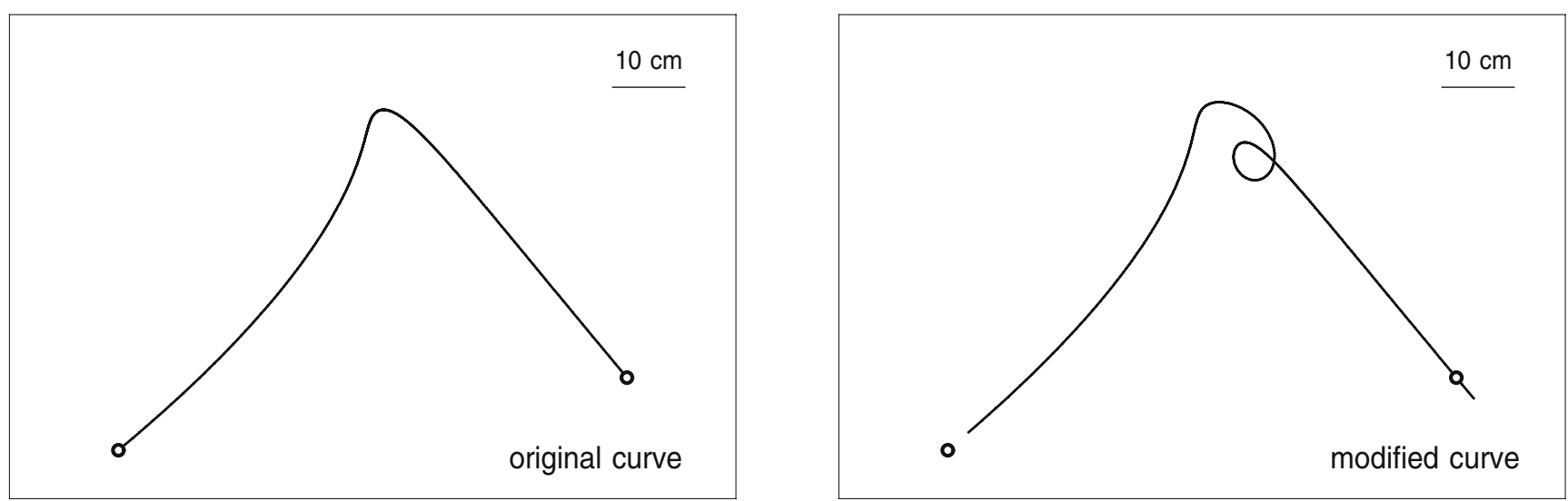

Fig. 3 Left: the PH quintic test curve for Example 1. Right: the modified curve defined by Eq. 11, which compensates for the machine/controller dynamics

Correspondingly, the parametric speed $\sigma(\xi)$ is a quartic polynomial with the Bernstein coefficients

$\sigma_{0}=\left|\mathbf{w}_{0}\right|^{2}, \quad \sigma_{1}=\operatorname{Re}\left(\mathbf{w}_{0} \overline{\mathbf{w}}_{1}\right)$

$\sigma_{2}=\frac{1}{3}\left[2\left|\mathbf{w}_{1}\right|^{2}+\operatorname{Re}\left(\mathbf{w}_{2} \overline{\mathbf{w}}_{0}\right)\right]$,

$\sigma_{3}=\operatorname{Re}\left(\mathbf{w}_{1} \overline{\mathbf{w}}_{2}\right), \quad \sigma_{4}=\left|\mathbf{w}_{2}\right|^{2}$,

and the arc length $s(\xi)$ is the quintic polynomial with Bernstein coefficients

$s_{0}=0 \quad$ and $\quad s_{k}=\frac{1}{5} \sum_{j=0}^{k-1} \sigma_{j} \quad$ for $k=1, \ldots, 5$.

The total arc length of this curve is $S=s(1)=s_{5}=$ $1.108098 \mathrm{~m}$.

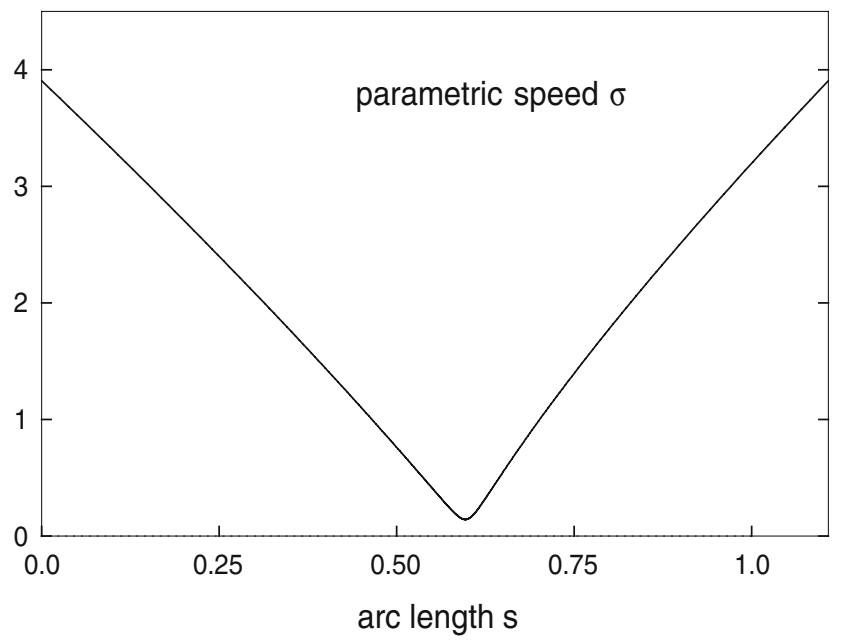

Figure 4 plots the parametric speed $\sigma$ and curvature $\kappa$ of this test curve, as functions of the arc length $s(\xi)$ rather than the parameter $\xi$. Because of the uneven parameter flow along the curve, plotting $\sigma$ and $\kappa$ as functions of $\xi$ fails to adequately portray the severity of their variation, which challenges the ability of the controller to faithfully execute the commanded path.

The same physical parameters are assumed for both the $x$ and $y$ axes, namely, $k_{a}=8 \mathrm{~A} / \mathrm{V}, k_{t}=$ $0.5 \mathrm{~N} \mathrm{~m} / \mathrm{A}, r_{g}=0.002 \mathrm{~m} / \mathrm{rad}, J=0.01 \mathrm{~kg} \mathrm{~m}^{2}, B=$ $0.025 \mathrm{~kg} \mathrm{~m}^{2} / \mathrm{s}$, and $\mathrm{P}$ controller gain $k_{p}=10 \mathrm{~V} / \mathrm{m}$, so $K=k_{a} k_{t} r_{g}=0.008, b_{x}=b_{y}=J / K k_{p}=0.125$, and $c_{x}=c_{y}=B / K k_{p}=0.3125$. With these values, the closed-loop system is stable for all positive $k_{p}$ (it is over-damped for $k_{p}<1.953$ and under-damped for $\left.k_{p}>1.953\right)$. Also, we choose a constant feedrate $V=$ $0.12 \mathrm{~m} / \mathrm{s}$, giving a nominal traversal time $T=S / V \approx$ $9.23 \mathrm{~s}$.

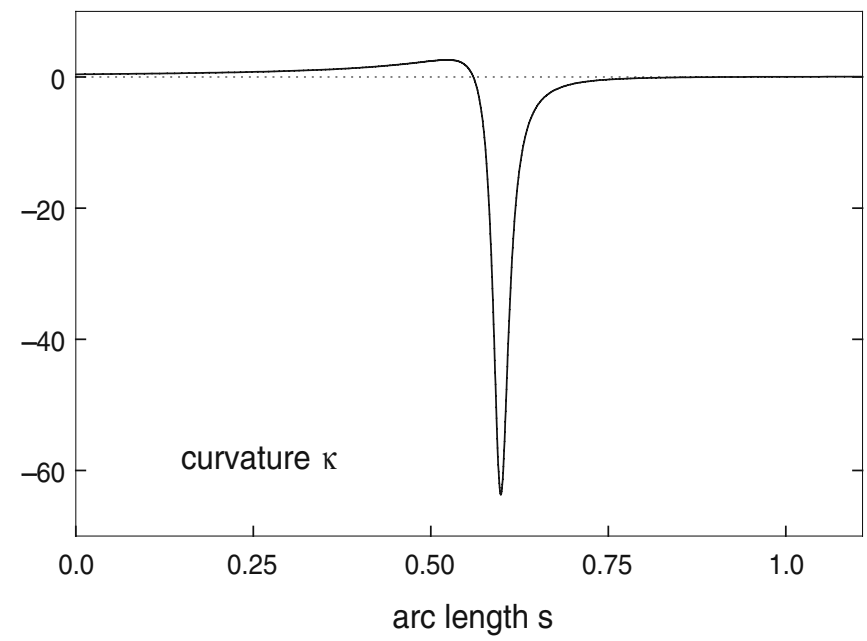

Fig. 4 The parametric speed $\sigma$ (left) and curvature $\kappa$ (right) of the PH quintic curve in Example 1, plotted as functions of the curve arc length $s$ 

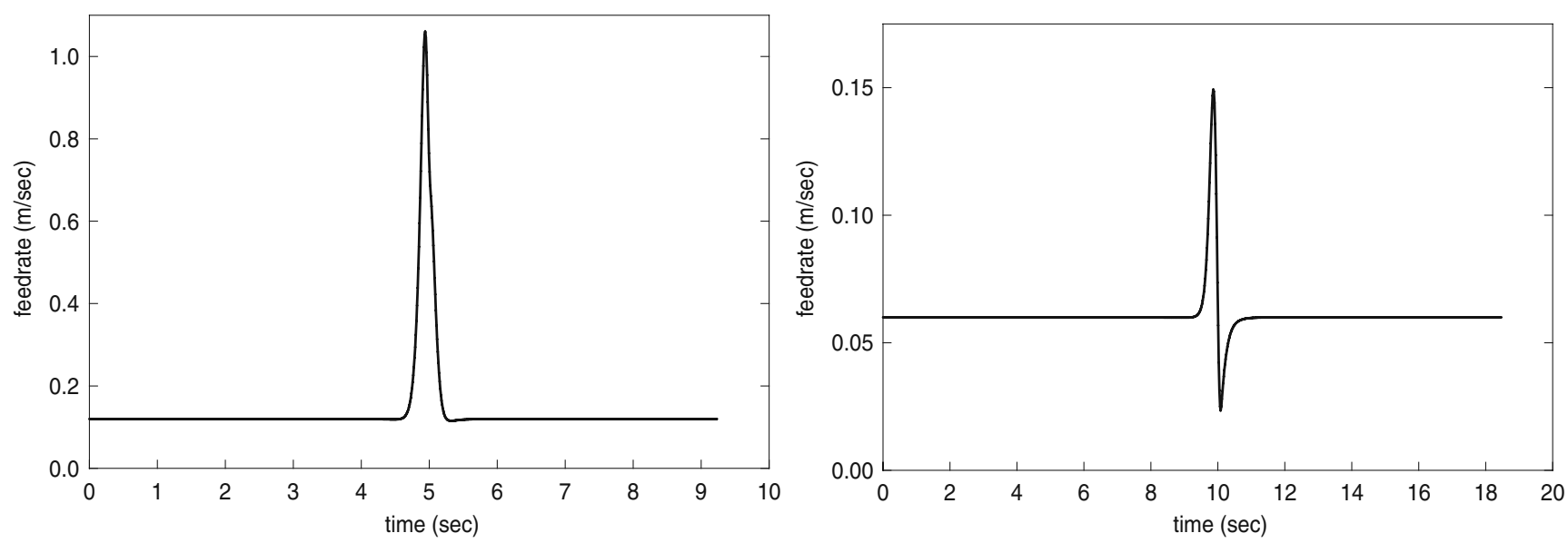

Fig. 5 Left: induced feedrate $\hat{V}$ along the modified path $\hat{\mathbf{R}}(\xi)$ in Fig. 3, in the case of a constant feedrate $V=0.12 \mathrm{~m} / \mathrm{s}$ along the original path $\mathbf{R}(\xi)$. Right: induced feedrate in the case $V=0.06 \mathrm{~m} / \mathrm{s}$ (note the different scales)

The parameter values were adopted (with slight modifications) from $[17,18]$. However, a much lower value of the gain $k_{p}$ has been chosen for illustrative purposes, to make the path errors and modifications plainly visible. For more realistic gains $k_{p}$, the path errors and modifications will be of much smaller magnitude. The chosen path contains a region of very high curvature, and it should be noted that the challenge this poses to the machine depends strongly on the feedrate, since the centripetal acceleration $\kappa V^{2}$ is proportional to the curvature and the square of the feedrate.

For the above parameters, Fig. 3 shows the modification of the commanded path defined by Eq. 11. A dramatic difference between the original and modified commanded paths is apparent near the region of high curvature. The peculiar rapid "looping" of the modified path is needed to compensate for the inability of the machine/controller dynamics to respond sufficiently fast to accurately track the sharply curved region of the original path. By means of this looping behavior, the modified path "tricks" the machine/controller into faithfully executing the original commanded path.

The arc length $\hat{S}=1.301524$ of the modified path exceeds that of the original path by $\sim 17.5 \%$ and, since the traversal time must be equal (see Section 3.3) to that for the unmodified path, the average induced feedrate on the modified path must be proportionately higher. When $b_{x}=b_{y}=b, c_{x}=c_{y}=c$, and $V=$ constant, one can express the derivative of $\hat{\mathbf{R}}(\xi)$ as

$$
\begin{aligned}
\hat{\mathbf{R}}^{\prime}= & {\left[1+b \frac{\left(3 \sigma^{\prime 2}-\sigma \sigma^{\prime \prime}\right) V^{2}}{\sigma^{4}}-c \frac{V \sigma^{\prime}}{\sigma^{2}}\right] \mathbf{R}^{\prime} } \\
& +\left[c-3 b \frac{V \sigma^{\prime}}{\sigma^{2}}\right] \mathbf{R}^{\prime \prime}+b \frac{V^{2}}{\sigma^{2}} \mathbf{R}^{\prime \prime \prime},
\end{aligned}
$$

and its parametric speed is $\hat{\sigma}=\left|\hat{\mathbf{R}}^{\prime}\right|$. As observed in Section 3.3, the induced feedrate along the modified path is $\hat{V}=(\hat{\sigma} / \sigma) V$, and this is shown in Fig. 5. $\hat{V}$ conforms closely to the nominal value $V=0.12 \mathrm{~m} / \mathrm{s}$ over most of $\hat{\mathbf{R}}(\xi)$ except the high curvature region, which incurs ${ }^{4}$ a ninefold "spike" in $\hat{V}$. This is a consequence of the fact that $\hat{\sigma}$ does not decrease as dramatically as $\sigma$, and hence, the ratio $\hat{\sigma} / \sigma$ becomes quite large. Figure 5 also shows the induced feedrate for the case $V=0.06 \mathrm{~m} / \mathrm{s}$.

For fixed values of all the other parameters, it is interesting to see how the modified path $\hat{\mathbf{R}}(\xi)$ depends on the constant feedrate $V$ and controller gain $k_{p}$. The effect of decreasing and increasing $V$ is illustrated in Fig. 6, while Fig. 7 shows that of decreasing and increasing $k_{p}$. For simplicity, a constant feedrate $V$ is employed in this example. As observed above, this implies that the modified path $\hat{\mathbf{R}}(\xi)$ does not coincide exactly with the original path $\mathbf{R}(\xi)$ at $\xi=0$ and 1 -this discrepancy is apparent in Figs. 3, 6, and 7.

Figure 8 compares commanded and executed trajectories (generated by the MATLAB simulations) for both the original path $\mathbf{R}(\xi)$ and the modified path $\hat{\mathbf{R}}(\xi)$ as input, and a fixed feedrate $V=0.12 \mathrm{~m} / \mathrm{s}$. For the original path, the executed trajectory deviates appreciably from the commanded path, due to the finite inertia and damping of the machine axes. When the modified path is used as input, on the other hand, the executed trajectory is essentially identical to the desired path $\mathbf{R}(\xi)$.

\footnotetext{
${ }^{4}$ This spike in feedrate, together with the looped path in Fig. 3, comprise the severe commanded behavior necessary to compensate for the machine inertia and damping, in executing the high-curvature region of the original curve at a fixed feedrate $V=0.12 \mathrm{~m} / \mathrm{s}$.
} 

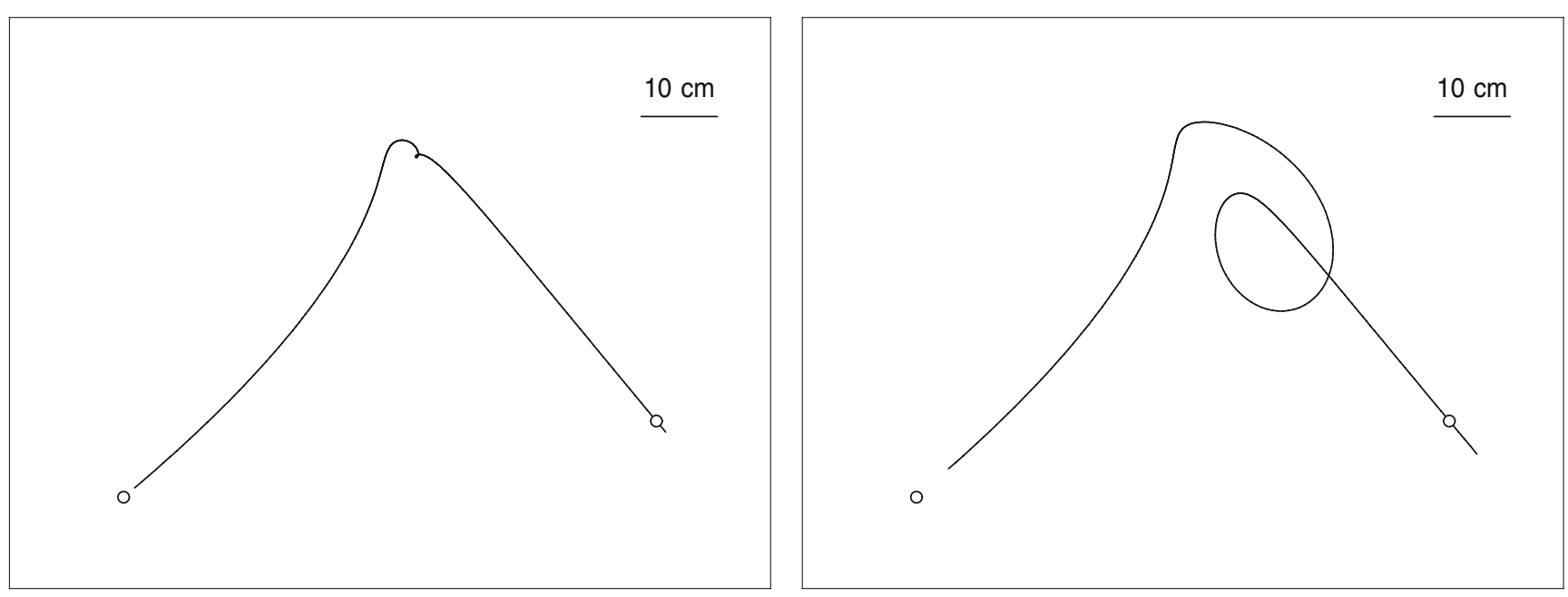

Fig. 6 Modified path for constant feedrates $V$ different from the nominal value $0.12 \mathrm{~m} / \mathrm{s}$ used in Fig. $3-$ left: $V=0.06 \mathrm{~m} / \mathrm{s}$, right: $V=0.18 \mathrm{~m} / \mathrm{s}$
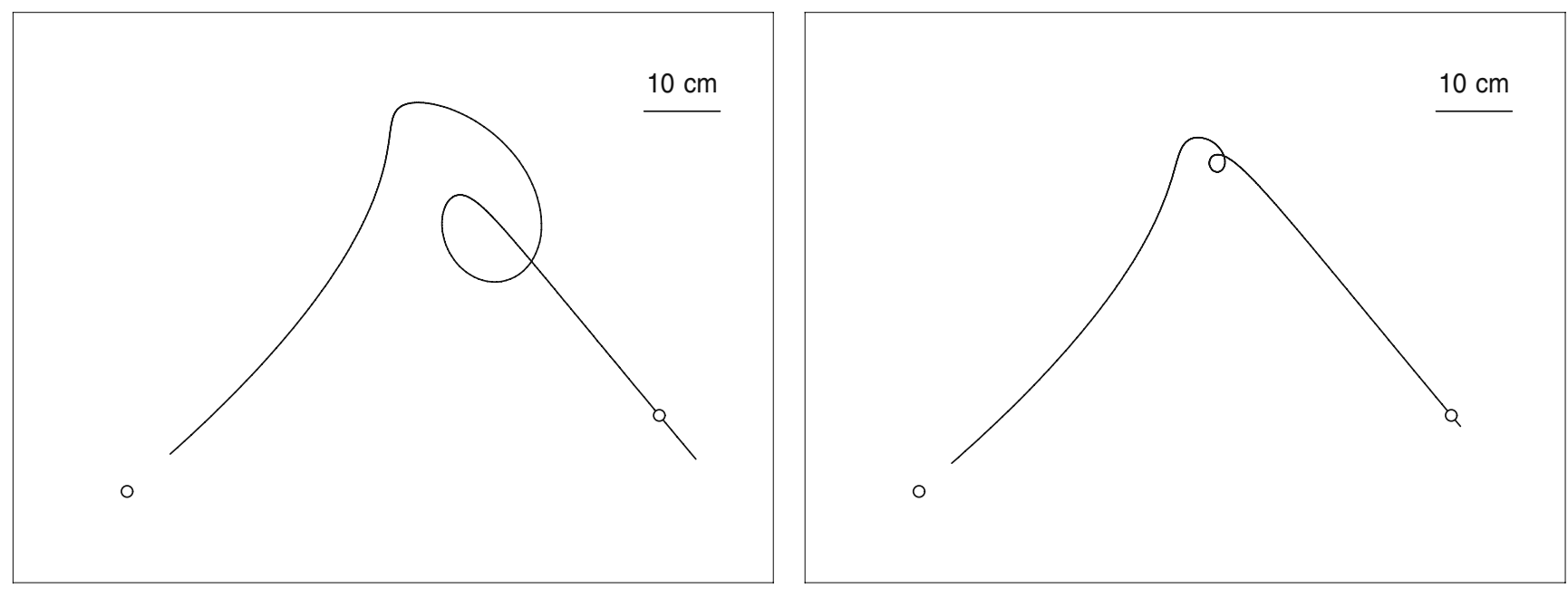

Fig. 7 Modified path for feedrate $V=0.12 \mathrm{~m} / \mathrm{s}$ and controller gains $k_{p}$ different from the nominal value 10 in Fig. 3-left: $k_{p}=5$, right: $k_{p}=20$

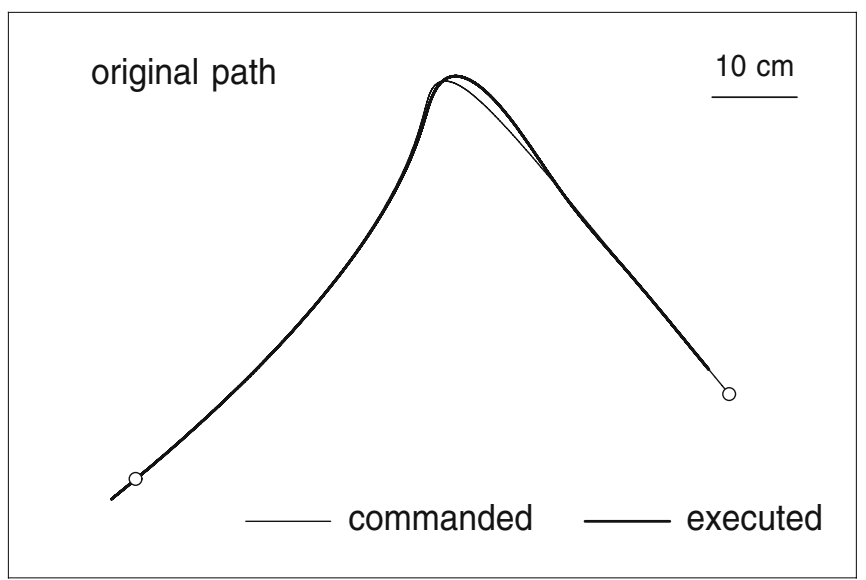

Fig. 8 Comparison of commanded and executed motions for original (left) and modified (right) paths. In the former case, the executed motion deviates significantly from the desired path.

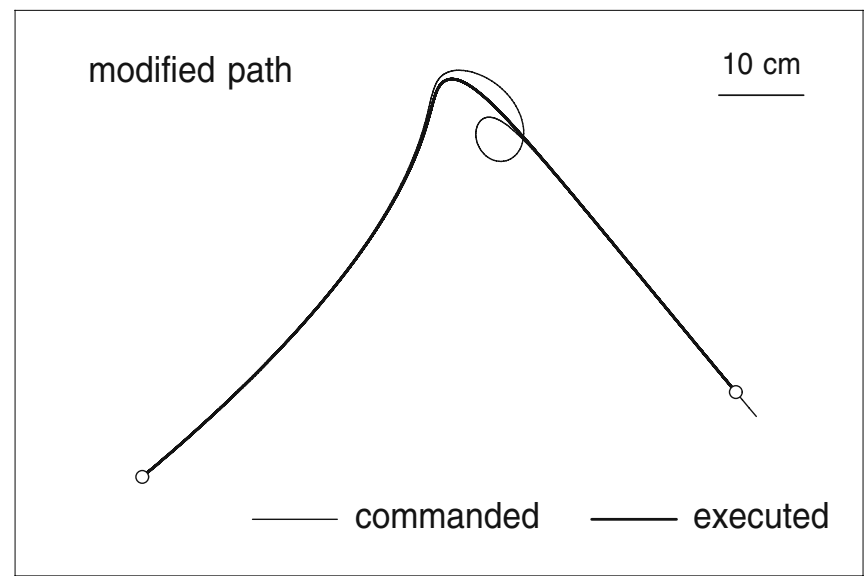

In the latter case, however, the executed motion is essentially indistinguishable from the original commanded path 

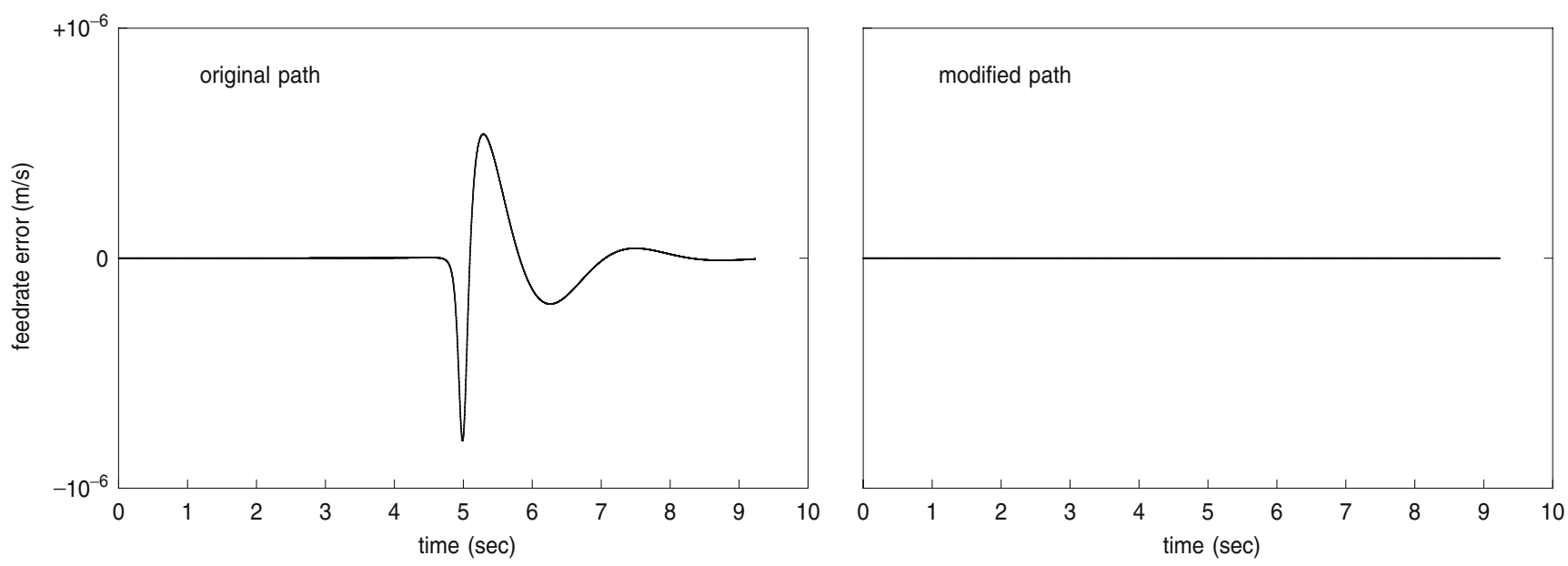

Fig. 9 Measured deviation (from MATLAB simulations) of actual feedrate about desired value $0.12 \mathrm{~m} / \mathrm{s}$ for original path (left) and modified path (right)

Furthermore, the modified path $\hat{\mathbf{R}}(\xi)$ and induced feedrate $^{5} \hat{V}(\xi)$ in combination yield an executed feedrate essentially identical to the desired constant feedrate $V=0.12 \mathrm{~m} / \mathrm{s}$. Figure 9 compares the actual feedrates for the original and modified paths, obtained from the MATLAB simulations. The feedrate fluctuations in the former case are rather small, and they are essentially eliminated using the modified path input.

The position error is defined, at each instant, as the distance between the actual machine position and the position along the specified path $\mathbf{R}(\xi)$ that corresponds to the prescribed feedrate $V$. Figure 10 illustrates the variation of position error obtained from the MATLAB simulations, for both the original and modified paths. This may be viewed as consisting of two componentsthe feed error in the tangent direction and the normal error orthogonal to it (taken as positive on the right of the curve tangent). As noted in Section 1, the feed error amounts to a timing discrepancy along the desired path, rather than a geometrical deviation from it. Figure 10 also shows the normal error, a better measure of the actual contour error -i.e., the geometrical distance between the desired and executed paths.

The maximum magnitude of the normal error is $0.0377 \mathrm{~m}$ for the original path and $0.000139 \mathrm{~m}$ for the modified path. Hence, the modified path gives a normal error more than two orders of magnitude smaller than the original path. In fact, when using the modified path as input, the normal error of the output path was found to decrease as the sampling frequency was increased,

\footnotetext{
${ }^{5}$ Note that $\hat{V}$ is generally non-constant, even when $V$ is constant (see Section 3.3).
}

indicating that it is primarily due to discretization of the input.

The Hausdorff distance [16] is a more rigorous measure of the difference between two curves. For arbitrary point sets $S_{1}, S_{2} \subset \mathbb{R}^{n}$, it is defined by

$\operatorname{distance}\left(S_{1}, S_{2}\right)=\max \left(\rho\left(S_{1}, S_{2}\right), \rho\left(S_{2}, S_{1}\right)\right)$,

where

$\rho\left(S_{j}, S_{k}\right)=\max _{\mathbf{p}_{j} \in S_{j}} \min _{\mathbf{p}_{k} \in S_{k}}\left|\mathbf{p}_{j}-\mathbf{p}_{k}\right|$.

The Hausdorff distance of the executed path from the desired path is found to be $0.0376 \mathrm{~m}$ for the original path, and $0.000006 \mathrm{~m}$ for the modified path.

Finally, Fig. 11 shows the $x$ and $y$ axis accelerations, using the original and modified paths. The acceleration magnitudes are evidently higher when using the modified path-as expected if the machine is required to traverse regions of strong curvature at high feedrates. In implementing modified paths on real CNC machines, one should verify a priori that such high accelerations will not exceed the torque capacity of the axis drive motors.

\subsection{PI controller}

For a PI controller, we have $d_{x}=d_{y}=0$ and $\left(c_{x}, c_{y}\right)=$ $\left(e_{x}, e_{y}\right)$, and by writing $(\hat{X}, \hat{Y})=(X, Y)+(\Delta X, \Delta Y)$ as before, Eq. 7 reduces to

$$
\begin{aligned}
& \mu_{x} \Delta X^{\prime}+v_{x} \Delta X=\alpha_{x} X^{\prime \prime \prime}+\beta_{x} X^{\prime \prime}+\tilde{\gamma}_{x} X^{\prime}, \\
& \mu_{y} \Delta Y^{\prime}+v_{y} \Delta Y=\alpha_{y} Y^{\prime \prime \prime}+\beta_{y} Y^{\prime \prime}+\tilde{\gamma}_{y} Y^{\prime},
\end{aligned}
$$

where $\tilde{\gamma}_{x}=V\left[\left(a_{x}\left(\sigma V^{\prime}-3 \sigma^{\prime} V\right)+b_{x} \sigma^{2}\right)\left(\sigma V^{\prime}-\sigma^{\prime} V\right)+\right.$ $\left.a_{x} \sigma V\left(\sigma V^{\prime \prime}-\sigma^{\prime \prime} V\right)\right]$ and $\tilde{\gamma}_{y}$ is analogously defined. 

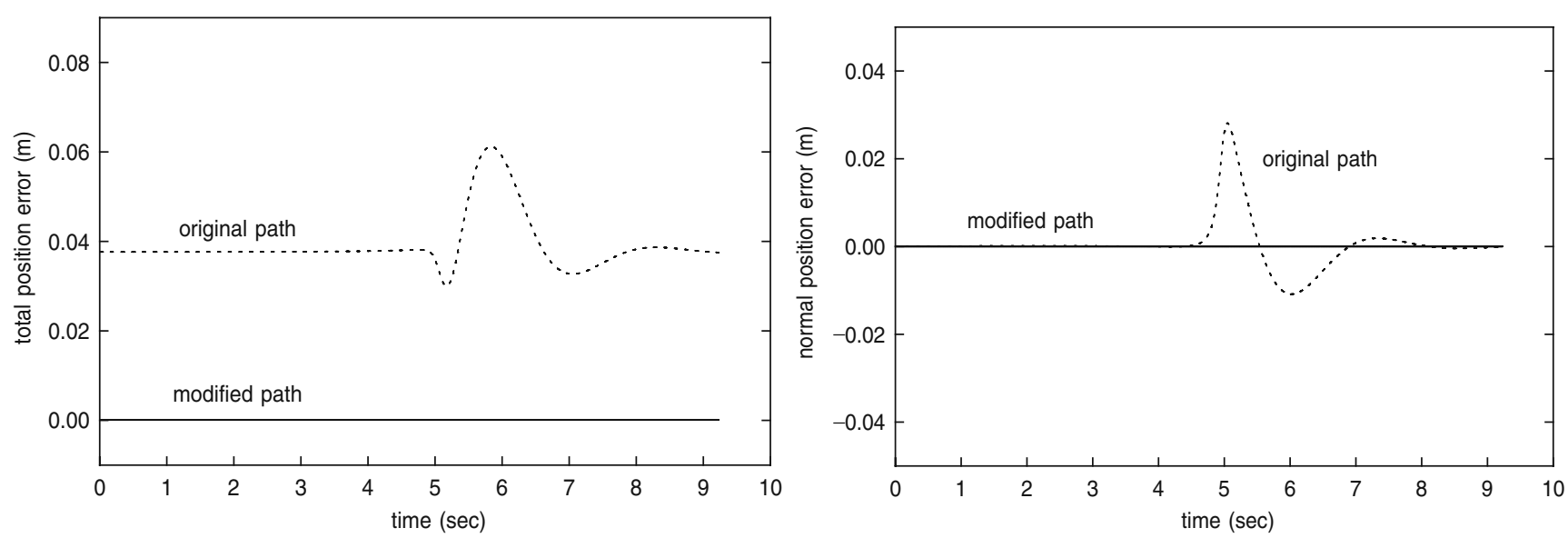

Fig. 10 Left: total position error (instantaneous distance between actual and desired position) from MATLAB simulations, for the original and modified paths. Right: Normal component of the total position error for these paths

These are linear, first-order differential equations for $\Delta X(\xi), \Delta Y(\xi)$ with polynomial coefficients. Since they do not ordinarily possess exact polynomial solutions (see the Appendix), we seek polynomial ${ }^{6}$ approximations of the solutions, expressed in the Bernstein basis on $[0,1]$. For brevity, we shall treat only the $x$ component of Eq. 13.

We denote the Bernstein basis of degree $n$ on $\xi \in$ $[0,1]$ by

$b_{i}^{n}(\xi)=\left(\begin{array}{c}n \\ i\end{array}\right)(1-\xi)^{n-i} \xi^{i}, \quad i=0, \ldots, n$,

and we define $b_{i}^{n}(\xi) \equiv 0$ when $i<0$ or $i>d$. If the commanded path $\mathbf{R}(\xi)$ is a degree- $n$ PH curve, its parametric speed $\sigma(\xi)$ and the feedrate function $V(\xi)$ can be expressed in Bernstein form as

$\sigma(\xi)=\sum_{i=0}^{n-1} \sigma_{i} b_{i}^{n-1}(\xi), \quad V(\xi)=\sum_{i=0}^{m} V_{i} b_{i}^{m}(\xi)$

Approximating the $x$-component of the displacement $\Delta \mathbf{R}(\xi)$ by a polynomial of degree $d$, we write

$$
\begin{aligned}
\Delta X(\xi) & =\sum_{i=0}^{d} \Delta X_{i} b_{i}^{d}(\xi), \\
\Delta X^{\prime}(\xi) & =\sum_{i=0}^{d-1} d\left(\Delta X_{i+1}-\Delta X_{i}\right) b_{i}^{d-1}(\xi) .
\end{aligned}
$$

Since the Eqs. 13 are first order, initial conditions must be specified for a unique solution. We take $\Delta X(0)=$

\footnotetext{
${ }^{6}$ Another possibility is to use piecewise-polynomial (spline) approximations, additional freedoms being obtained by increasing the number of knots rather than the degree.
}

$\Delta X_{0}=0$ and $\Delta Y(0)=\Delta Y_{0}=0$, so the modified path coincides with the original commanded path at $\xi=0$.

The polynomials in Eq. 6 are constructed in the Bernstein form on $\xi \in[0,1]$. The right-hand side of the $x$ component of Eq. 13 is then a known polynomial of degree $6 n-5$, which we denote $Q_{x}(\xi)$. We wish to choose $\Delta X_{1}, \ldots, \Delta X_{d}$ in Eq. 14 to make the left-hand side of Eq. 13 agree "as close as possible" to $Q_{x}(\xi)$.

A number of different methods, with associated measures for closeness of approximation, are possible. By expressing the left- and right-hand sides in the Chebyshev basis on $[0,1]$, for example, approximations that minimize the maximum error over that interval can be determined. Another approach is a least-squares minimization of the expression

$$
\begin{aligned}
F\left(\Delta X_{1}, \ldots, \Delta X_{d}\right)= & \int_{0}^{1}\left[\mu_{x}(\xi) \Delta X^{\prime}(\xi)\right. \\
& \left.+v_{x}(\xi) \Delta X(\xi)-Q_{x}(\xi)\right]^{2} \mathrm{~d} \xi,
\end{aligned}
$$

yielding a system of linear equations for $\Delta X_{1}, \ldots$, $\Delta X_{d}$. Or for suitable nodes $\xi_{1}, \ldots, \xi_{d}$, one can obtain these coefficients through an interpolatory process, requiring the left-hand side to agree in value with $Q_{x}(\xi)$ at the nodes.

Such methods incur important considerations regarding (1) the condition (sensitivity) of the coefficients with respect to changes in the input data, (2) the convergence behavior of the approximants, (3) the ability to ensure that the approximants satisfy a prescribed tolerance, and (4) computational cost of the method. A detailed analysis/comparison of all these factors is beyond our present scope, and we defer it to a later study. At present, we are content to employ a simple approximation scheme to illustrate the path 

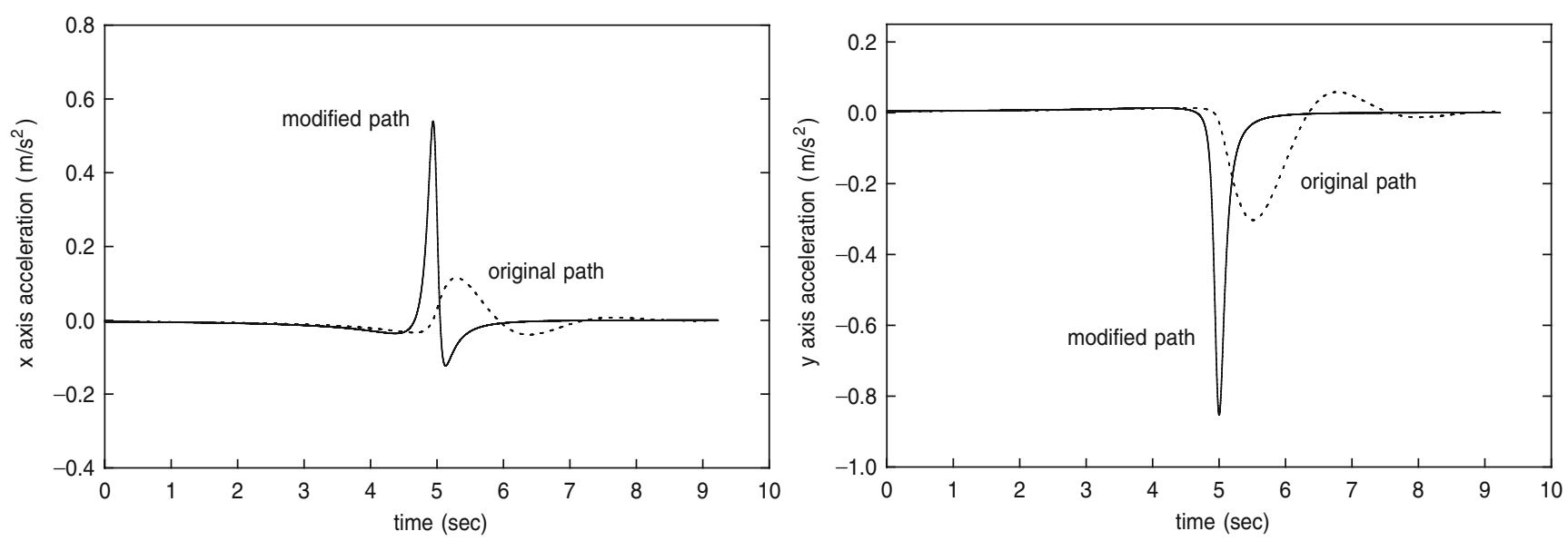

Fig. 11 Left: $x$-axis acceleration from MATLAB simulations for original and modified paths. Right: $y$-axis acceleration for original and modified paths

modification for a PI controller, based on choosing the coefficients $\Delta X_{1}, \ldots, \Delta X_{d}$ so that the left-hand side of Eq. 13 agrees with $Q_{x}(\xi)$ at the $d$ Chebyshev nodes

$\xi_{k}=\frac{1}{2}\left[1-\cos \left(\frac{k \pi}{d-1}\right)\right], \quad k=0, \ldots, d-1$.

It is well-known [3] that interpolation at the Chebyshev nodes suppresses the spurious oscillations that interpolants on uniform nodes are susceptible to. To avoid numerical stability issues, the linear equations for $\Delta X_{1}, \ldots, \Delta X_{d}$ were constructed and solved in the MAPLE computer algebra system.

Example 2 For the PI controller, the $\mathrm{PH}$ quintic curve $\mathbf{R}(\xi)$ of Example 1 is chosen as the commanded path,

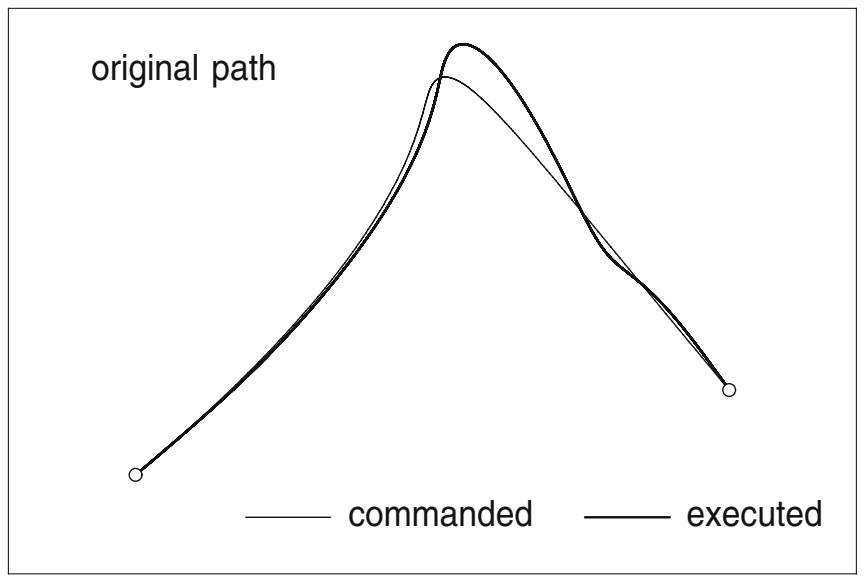

Fig. 12 Commanded and executed motions for original (left) and modified (right) paths, using the PI controller-compare with $\mathrm{P}$ controller motions in Fig. 8. With the original path, the exe- again with a constant feedrate $V=0.12 \mathrm{~m} / \mathrm{s}$ and the same physical parameters as before, and $k_{p}=k_{i}=10$ as the $\mathrm{P}$ and I controller gains. For the chosen parameters, the closed-loop system is stable with poles at -1.242708 and $-0.628646 \pm 2.458121$.

Note that the Chebyshev nodes (Eq. 15) are more densely spaced at the ends of the interval $\xi \in[0,1]$ than at the center, where the high-curvature region of the path occurs. To ensure accurate approximation of the modified path, we choose a relatively high approximant degree, $d=30$.

Figure 12 compares the commanded and executed motions obtained with the original and modified paths, using the PI controller (it is interesting to compare these with the results from the P controller-see Fig. 8). Using the original path, the executed motion exhibits

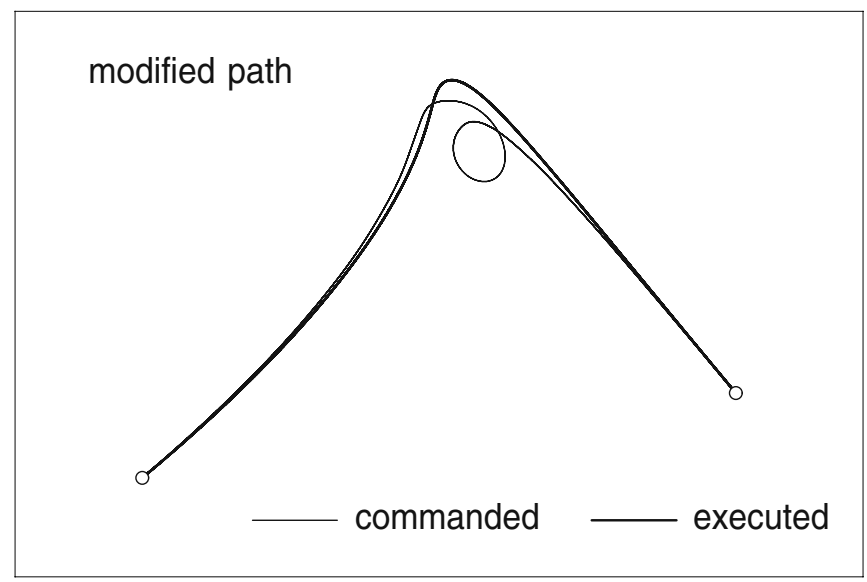

cuted motion deviates significantly from the desired path. With the modified path, however, the executed motion is essentially indistinguishable from the original commanded path 

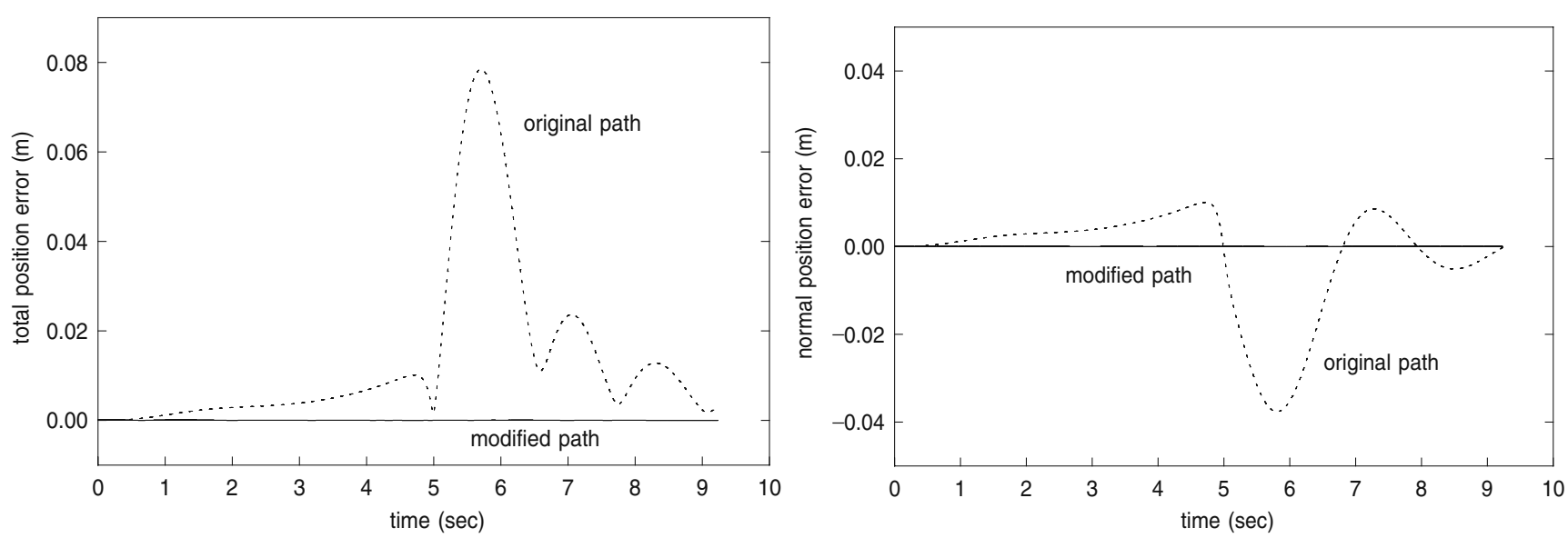

Fig. 13 Measured path error (from MATLAB simulations) for the original and modified paths using a PI controller with gains $k_{p}=k_{i}=$ 10 and feedrate $V=0.12 \mathrm{~m} / \mathrm{s}$ - left: total position error, right: normal position error

a very significant deviation from the commanded path, although the integrator in the controller allows the machine to rapidly "recover" from this deviation after it passes the high-curvature region. Using the modified path, on the other hand, the executed motion corresponds to an accurate traversal of the original commanded path, at the commanded feedrate $V=$ $0.12 \mathrm{~m} / \mathrm{s}$.

Figure 13 compares the total and normal path errors for the original and modified paths obtained with the PI controller. Note that the adopted initial conditions $\Delta X(0)=\Delta Y(0)=0$ for the differential equations (Eq. 13) guarantee zero error at the start of the simulation. Also, the presence of an integrator in the controller transfer function effectively suppresses the error for the original path after a while. The normal error results are qualitatively similar to those obtained with the P controller: for the original path, the maximum normal error is of similar magnitude (but opposite sign); for the modified path, the normal error is essentially indistinguishable from measurement noise (caused by discretization of the input).

Figure 14 compares the $x$ and $y$ axis accelerations along the original and modified paths obtained with the PI controller. Because of the new controller transfer function, the accelerations along the original path differ from those for the $\mathrm{P}$ controller. On the other hand, the $x$ and $y$ axis accelerations along the modified path obtained with the P and the PI controller are essentially identical, since in both cases the modified path effectively compensates for the machine/controller dynamics to yield an output motion corresponding to accurate traversal of the original path at the specified $V=0.12 \mathrm{~m} / \mathrm{s}$ feedrate.
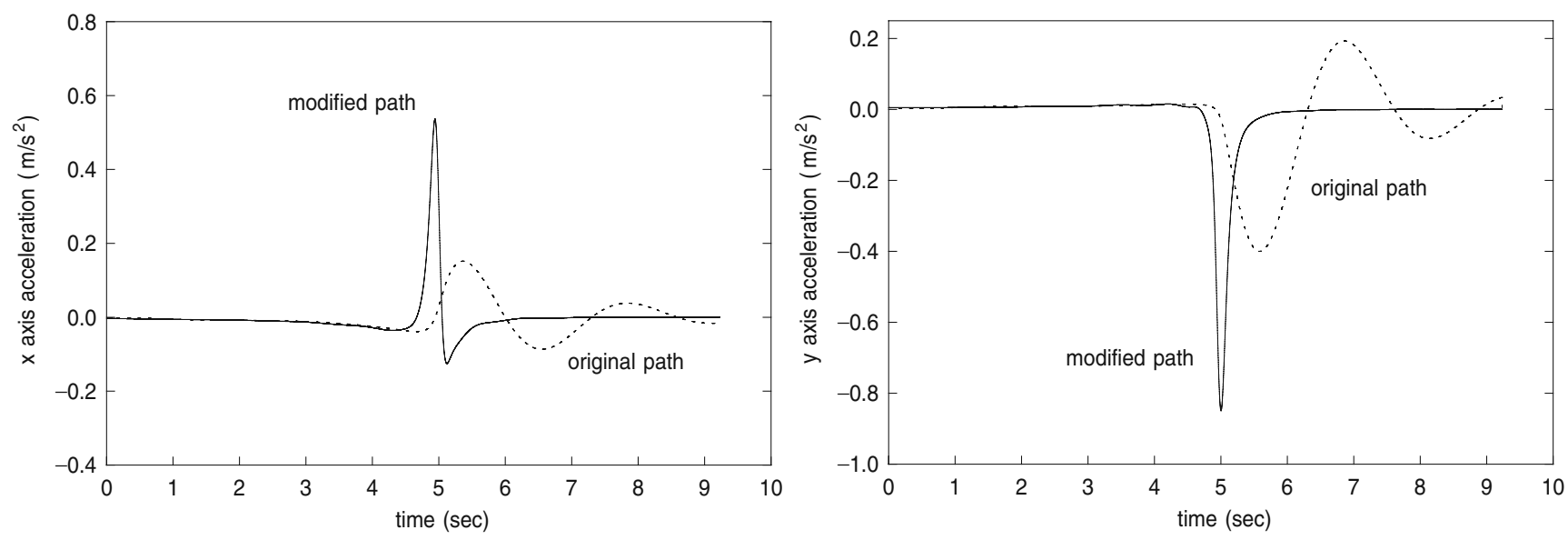

Fig. 14 Axis accelerations (from MATLAB simulations) for the original and modified paths when using a PI controller with gains $k_{p}=$ $k_{i}=10$ and feedrate $V=0.12 \mathrm{~m} / \mathrm{s}$ - left: $x$-axis acceleration, right: $y$-axis acceleration 
The above approach also applies in the case of a PID controller, differing in the details for the computation of the coefficients of $\Delta X(\xi), \Delta Y(\xi)$ but not in the basic methodology.

\section{Closure}

A novel approach to compensating for contour errors incurred by the inherent dynamic limitations of CNC machine/controller systems has been introduced, based on computing an a priori modification $\hat{\mathbf{R}}(\xi)$ of the commanded path $\mathbf{R}(\xi)$. For a specified feedrate variation $V(\xi)$, the independent variable in the dynamical equations is first transformed from time $t$ to the curve parameter $\xi$. By reverting the equations (i.e., swapping the input/output roles) a modified path $\hat{\mathbf{R}}(\xi)$ that-modulated by the machine/controller dynamics-exactly yields the desired path $\mathbf{R}(\xi)$ as the physical output, can be characterized as the solution of linear differential equations with polynomial coefficients.

In the case of standard second-order models for machine axis dynamics, a P-type controller, and a $\mathrm{PH}$ curve as the original commanded path $\mathbf{R}(\xi)$, the modified path $\hat{\mathbf{R}}(\xi)$ can be exactly described as a higherorder rational curve. Exact closed-form solutions are not possible for more sophisticated controller types, but accurate polynomial approximations can be readily determined through interpolatory or least-squares procedures. These methods will be fully developed and investigated in subsequent papers.

The accuracy achievable in machining operations is, of course, dependent on many factors other than inertia and damping of the axes (cutting forces, machine rigidity, backlash in the drive system, etc.). The methods described herein may also be capable of compensating for such effects, if quantitative models of them are available. In practice, the relative magnitude of errors due to machine dynamics, cutting forces, etc., will depend on many technical factors (machine architecture, cutting speeds and feedrates, material being cut, cutting tool, etc.) and must be assessed on a case-by-case basis.

The intent of this paper was to describe the fundamental methodology of the inverse-dynamics pathmodification procedure, and to demonstrate its efficacy in reducing contour error through illustrative examples involving P or PI controllers and constant feedrates along paths with strong variations of curvature or parametric speed. More detailed results concerning the practical implementation, experimental performance analysis, and optimal choice of control parameters for the method will be presented in due course.
Open Access This article is distributed under the terms of the Creative Commons Attribution Noncommercial License which permits any noncommercial use, distribution, and reproduction in any medium, provided the original author(s) and source are credited.

\section{Appendix: ODEs with polynomial coefficients}

When the machine/controller dynamical equations are transformed from the time $t$ to the curve parameter $\xi$ as the independent variable, their coefficients become polynomials in $\xi$ rather than constants. Although all derivatives of a polynomial function are polynomials of lower degree, differential equations with polynomial coefficients do not (in general) possess polynomial solutions. Since this fact is of fundamental importance to the inverse dynamics problem, but not well-known, we now briefly review from first principles the restricted conditions under which polynomial solutions may exist.

Consider an inhomogeneous linear differential equation of the form

$a_{r}(x) \frac{\mathrm{d}^{r} y}{\mathrm{~d} x^{r}}+\cdots+a_{1}(x) \frac{\mathrm{d} y}{\mathrm{~d} x}+a_{0}(x) y=b(x)$,

where $a_{r}(x), \ldots, a_{0}(x)$ and $b(x)$ are polynomials. To see why this does not (in general) admit a polynomial solution $y(x), \operatorname{let} \operatorname{deg}\left(a_{k}\right)=\ell_{k}$ for $k=0, \ldots, r, \operatorname{deg}(b)=$ $m$, and $y(x)=c_{0}+c_{1} x+\cdots+c_{n} x^{n}$. Recall [2] that the most general solution of Eq. 16 is expressed in terms of arbitrary constants $\lambda_{1}, \ldots, \lambda_{r}$ as

$y(x)=\lambda_{1} y_{1}(x)+\cdots+\lambda_{r} y_{r}(x)+y_{p}(x)$,

where $y_{1}(x), \ldots, y_{r}(x)$ are linearly independent solutions of the homogeneous equation, and $y_{p}(x)$ is a particular solution of the inhomogeneous equation. Consider first the homogeneous equation, corresponding to $b(x) \equiv 0$ in Eq. 16. If $y(x)=c_{0}+c_{1} x+\cdots+$ $c_{n} x^{n}$, the left-hand side is a polynomial of degree

$d=n+\max _{0 \leq k \leq r}\left(\ell_{k}-k\right)$

in $x$, and clearly $d \geq n$. To satisfy the homogeneous equation, this polynomial must vanish identically. This corresponds to requiring the $n+1$ coefficients $c_{0}, \ldots, c_{n}$ of $y(x)$ to satisfy $d+1$ homogeneous linear equations (where $d \geq n$ ). For a non-trivial solution, $\left(c_{0}, \ldots, c_{n}\right) \neq(0, \ldots, 0)$, the matrix of this linear system-with entries defined by the coefficients of $a_{0}(x), \ldots, a_{r}(x)$-must be of rank $<n+1$. Since this deficiency in rank is only achieved for special choices of the polynomials $a_{0}(x), \ldots, a_{r}(x)$, the solutions $y_{1}(x), \ldots, y_{r}(x)$ of the homogeneous equation are not, in general, polynomials in $x$. 
Consider now the inhomogeneous equation. The degree (Eq. 17) of the left-hand side of Eq. 16 with $y(x)=c_{0}+c_{1} x+\cdots+c_{n} x^{n}$ must agree with the degree of $b(x)$-i.e., we must have

$m=n+\max _{0 \leq k \leq r}\left(\ell_{k}-k\right)$.

If this is satisfied, the differential equation (Eq. 16) reduces to a system of $m+1$ linear equations for the $n+1$ coefficients $c_{0}, \ldots, c_{n}$ of $y(x)$. Clearly, we must have $m \leq n$ and hence $\ell_{k} \leq k$ for $k=0, \ldots, r$ if these equations are not to be over-determined. Specifically, this implies that Eq. 16 does not (in general) have a polynomial solution $y(x)$ if $a_{0}(x), a_{1}(x), a_{2}(x), \ldots$ are of higher degree than constant, linear, quadratic, ... in $x$. Hence, the inhomogeneous equation does not admit polynomial solutions for general choices of $a_{0}(x), \ldots, a_{r}(x)$.

\section{References}

1. Altintas Y (2000) Manufacturing automation: metal cutting mechanics, machine tool vibrations, and $\mathrm{CNC}$ design. Cambridge University Press, Cambridge

2. Coddington EA (1961) An introduction to ordinary differential equations. Dover, New York (reprint)

3. Davis PJ (1975) Interpolation and approximation. Dover, New York (reprint)

4. de Souza AF, Coelho RT (2007) Experimental investigation of feed rate limitations on high speed milling aimed at industrial applications. Int J Adv Manuf Technol 32:1104-1114

5. Erkorkmaz K, Yeung C-H, Altintas Y (2006) Virtual CNC system. Part II. High speed contouring application. Int J Mach Tools Manuf 46:1124-1138

6. Farouki RT (1994) The conformal map $z \rightarrow z^{2}$ of the hodograph plane. Comput Aided Geom Des 11:363-390

7. Farouki RT (2008) Pythagorean-hodograph curves: algebra and geometry inseparable. Springer, Berlin

8. Farouki RT, Goodman TNT (1996) On the optimal stability of the Bernstein basis. Math Comput 65:1553-1566
9. Farouki RT, Neff CA (1995) Hermite interpolation by Pythagorean-hodograph quintics. Math Comput 64:15891609

10. Farouki RT, Rajan VT (1987) On the numerical condition of polynomials in Bernstein form. Comput Aided Geom Des 4:191-216

11. Farouki RT, Rajan VT (1988) Algorithms for polynomials in Bernstein form. Comput Aided Geom Des 5:1-26

12. Farouki RT, Sakkalis T (1991) Real rational curves are not "unit speed." Comput Aided Geom Des 8:151-157

13. Farouki RT, Sakkalis T (2007) Rational space curves are not "unit speed." Comput Aided Geom Des 24:238-240

14. Farouki RT, Shah S (1996) Real-time CNC interpolators for Pythagorean-hodograph curves. Comput Aided Geom Des 13:583-600

15. Farouki RT, Tsai Y-F (2001) Exact Taylor series coefficients for variable-feedrate CNC curve interpolators. Comput Aided Des 33:155-165

16. Hausdorff F (1957) Set theory (translated by J. R. Aumann et al.). Chelsea, New York

17. Imani BM, Jahanpour J (2008) High-speed contouring enhanced with PH curves. Int J Adv Manuf Technol 37:747-759

18. Jahanpour J, Imani BM (2008) Real-time PH curve CNC interpolators for high speed cornering. Int J Adv Manuf Technol 39:302-316

19. Komanduri R, Subramanian K, von Turkovich BF (eds) (1984) High speed machining. PED, vol 12. ASME, New York

20. Shpitalni M, Koren Y, Lo CC (1994) Realtime curve interpolators. Comput Aided Des 26:832-838

21. Smith S, Tlusty J (1997) Current trends in high-speed machining. ASME J Manuf Sci Eng 119:664-666

22. Tlusty J (1993) High-speed machining. CIRP Ann 42:733-738

23. Tsai Y-F, Farouki RT (2001) Algorithm 812: BPOLY: an object-oriented library of numerical algorithms for polynomials in Bernstein form. ACM Trans Math Softw 27:267-296

24. Yang DCH, Kong T (1994) Parametric interpolator versus linear interpolator for precision $\mathrm{CNC}$ machining. Comput Aided Des 26:225-234

25. Yeh S-S, Hsu P-L (1999) The speed-controlled interpolator for machining parametric curves. Comput Aided Des 31:349357

26. Yeung, C-H, Altintas Y, Erkorkmaz K (2006) Virtual CNC system. Part I. System architecture. Int J Mach Tools Manuf 46:1107-1123 